\title{
FITTING TRAFFIC TRACES WITH DISCRETE CANONICAL PHASE TYPE DISTRIBUTIONS AND MARKOV ARRIVAL PROCESSES
}

\author{
ANDRÁS MÉSZÁROS *,***, JÁNOS PAPP* MiKLÓS TELEK *,** \\ * Department of Networked Systems and Services \\ Budapest University of Technology and Economics, Magyar tudósok krt 2, 1117 Budapest, Hungary \\ e-mail: (meszarosa, telek) ahit.bme.hu, papp.janos.90@gmail.com \\ ** MTA-BME Information Systems Research Group \\ Magyar tudósok krt. 2, 1117 Budapest, Hungary \\ *** Inter-University Center for Telecommunications and Informatics \\ Kassai út 26, 4028 Debrecen, Hungary
}

\begin{abstract}
Recent developments of matrix analytic methods make phase type distributions (PHs) and Markov Arrival Processes (MAPs) promising stochastic model candidates for capturing traffic trace behaviour and for efficient usage in queueing analysis. After introducing basics of these sets of stochastic models, the paper discusses the following subjects in detail: (i) PHs and MAPs have different representations. For efficient use of these models, sparse (defined by a minimal number of parameters) and unique representations of discrete time PHs and MAPs are needed, which are commonly referred to as canonical representations. The paper presents new results on the canonical representation of discrete PHs and MAPs. (ii) The canonical representation allows a direct mapping between experimental moments and the stochastic models, referred to as moment matching. Explicit procedures are provided for this mapping. (iii) Moment matching is not always the best way to model the behavior of traffic traces. Model fitting based on appropriately chosen distance measures might result in better performing stochastic models. We also demonstrate the efficiency of fitting procedures with experimental results.
\end{abstract}

Keywords: fitting traffic traces, discrete phase type distribution, discrete Markov arrival process, canonical representation.

\section{Introduction}

Stochastic models with underlying Markov chains are known for being flexible in modelling general stochastic behaviour and for allowing efficient numerical analysis through matrix analytic methods (Neuts, 1981). These nice properties make phase type distributions (PHs) and Markov Arrival Processes (MAPs) promising candidates for modelling the traffic load of computer and communication systems.

For a period of time, continuous time stochastic models were more often applied in performance modelling of computer and communication systems. Later on, with the rise of slotted time telecommunication protocols (e.g., ATM), discrete time models became primary modelling tools (for recent surveys, see Alfa, 2002; Lakatos et al., 2013). In this paper we focus on discrete time models and present some results whose continuous time counterparts are already available. It turns out that discrete time models with strictly positive eigenvalues are practically identical with their continuous time counterparts, but discrete time models containing also negative eigenvalues pose new problems.

One main problem of PHs and MAPs is the non-uniqueness and over-parametrization of their general matrix form (see, e.g., the work of Telek and Horváth (2007) for more details). Specifically, there are descriptions with a minimal number of parameters for describing these processes, but those descriptions are hard to use in practice because they do not indicate the feasibility of the associated stochastic model (for example, the moments of a random variable of a given class might define the random variable fully, but it is not easy to check if a set of moments is feasible, i.e., if there exists a random variable in the given class with those moments). On the other hand, over-parametrised matrix descriptions give a direct mapping to Markov chains, 
which ensures the feasibility of the model. However, the over-parametrization causes significant problems in fitting methods. The above obstacle can be eliminated by finding unique matrix representations with a minimal number of parameters. These representations are referred to as canonical representations. Apart from the benefits in fitting methods, canonical representations also enable parameter matching, i.e., a direct mapping of important traffic parameters (moments, autocorrelation) to these models.

In this paper we present new results on the canonical representation of second- and third-order discrete PHs (DPH(2) and DPH(3), respectively) as well as on second-order discrete MAPs (DMAP(2)). We provide explicit formulae for parameter matching using these canonical forms, give moments and correlation bounds for these models, and show their efficiency in fitting through numerical examples.

The rest of the paper is organized as follows. In Section 2 we survey the necessary definitions and essential properties of existing Markov chain driven stochastic processes and their non-Markovian generalizations. The discussion of canonical forms for $\mathrm{DPH}(2), \operatorname{DPH}(3)$ and DMAP(2) can be found in Sections 3, 4, and 5, respectively. Section 2 gives formulae for parameter matching. Section 6 presents moments based matching methods for approximating discrete PHs and MAPs. The numerical examples for trace fitting are presented in Section 7. Section 8 concludes the paper.

\section{Markov chain driven point processes and their non-Markovian generalizations}

The following subsections summarize the main properties of simple stochastic models with a background discrete state Markov chain and their non-Markovian generalizations. If the background chain is a discrete time Markov chain, we obtain discrete (time) stochastic models, and if it is a continuous time Markov chain, we obtain continuous (time) stochastic models. The main focus of the paper is on discrete models, but some results are related to their continuous counterparts. Accordingly, we introduce both of them.

2.1. Discrete phase type and matrix geometric distributions. The following stochastic models define discrete distributions on the positive integers.

Definition 1. Let $\mathcal{X}$ be a discrete random variable on $\mathbb{N}^{+}$ with probability mass function (pmf)

$$
\begin{aligned}
P_{\mathcal{X}}(i) & =\operatorname{Pr}(\mathcal{X}=i) \\
& =\alpha \boldsymbol{A}^{i-1}(\mathbb{1}-\boldsymbol{A} \mathbb{1}), \quad \forall i \in \mathbb{N}^{+},
\end{aligned}
$$

where $\alpha$ is a row vector of size $n, \boldsymbol{A}$ is a square matrix of size $n \times n$, and $\mathbb{1}$ is the column vector of ones of size $n$. If the pmf has this matrix geometric form, then we say that $\mathcal{X}$ is a matrix geometrically distributed with representation $(\alpha, \boldsymbol{A})$ or, briefly, $\operatorname{MG}(\alpha, \boldsymbol{A})$ distributed.

The size of $\boldsymbol{A}$ is also referred to as the order of the associated distribution. In this and the subsequent models, scalar quantities are obtained as a product of a row vector, a given number of square matrices and a column vector. In the sequel, we refer to the row vector as an initial vector and to the column vector as a closing vector. It is an important consequence of Definition 1 that $\alpha$ and $\boldsymbol{A}$ have to be such that (1) is non-negative.

Definition 2. If $\mathcal{X}$ is an $\operatorname{MG}(\alpha, \boldsymbol{A})$ distributed random variable, where $\alpha$ and $\boldsymbol{A}$ have the following properties:

$$
\begin{aligned}
& \text { - } \alpha_{i} \geq 0, \\
& \text { - } A_{i j} \geq 0, \boldsymbol{A} \mathbb{1} \leq \mathbb{1},
\end{aligned}
$$

then we say that $\mathcal{X}$ is discrete phase type distributed with representation $(\alpha, \boldsymbol{A})$ or, shortly, $\operatorname{DPH}(\alpha, \boldsymbol{A})$ distributed.

The vector-matrix representations satisfying the conditions of Definition 2 are called Markovian.

In this paper we focus on distributions on the positive integers. Consequently, $\alpha \mathbb{1}=1$. The cumulative density function (cdf), the moment generating function, and the factorial moments of $\mathcal{X}$ are

$$
\begin{aligned}
F_{\mathcal{X}}(i) & =\operatorname{Pr}(\mathcal{X} \leq i)=1-\alpha \boldsymbol{A}^{i} \mathbb{1} \\
f_{n} & =E(\mathcal{X}(\mathcal{X}-1) \ldots(\mathcal{X}-n+1)) \\
& =n ! \alpha(\boldsymbol{I}-\boldsymbol{A})^{-n} \boldsymbol{A}^{n-1} \mathbb{1} .
\end{aligned}
$$

A DPH has infinitely many different Markovian and non-Markovian representations (matrix-vector pairs, which fulfill (1)). One way to get a different representation of a $\operatorname{DPH}(\boldsymbol{\alpha}, \boldsymbol{A})$ with the same size is the application of the similarity transformation

$$
\boldsymbol{B}=\boldsymbol{T}^{-1} \boldsymbol{A T}, \quad \beta=\alpha \boldsymbol{T},
$$

where $\boldsymbol{T}$ is an arbitrary non-singular matrix for which $\boldsymbol{T} \mathbb{1}=\mathbb{1}$. If a DPH has an $(\alpha, \boldsymbol{A})$ Markovian representation, for which $\boldsymbol{A}$ is upper triangular, we call the distribution acyclic DPH (shortly ADPH) distribution, and the specific representation an ADPH representation.

2.2. Discrete Markov arrival processes and discrete rational arrival processes. Let $\mathcal{X}(t)$ be a point process on $\mathbb{N}^{+}$with a joint probability mass function of inter-event times $P_{\mathcal{X}(t)}\left(x_{0}, x_{1}, \ldots, x_{k}\right)$ for $k=1,2, \ldots$ and $x_{0}, \ldots, x_{k} \in \mathbb{N}^{+}$.

Definition 3. $\mathcal{X}(t)$ is called a rational arrival process if there exists a finite $\left(\boldsymbol{H}_{\mathbf{0}}, \boldsymbol{H}_{\mathbf{1}}\right)$ square matrix pair such that $\left(\boldsymbol{H}_{0}+\boldsymbol{H}_{1}\right) \mathbb{1}=\mathbb{1}$,

$$
\underline{\pi}\left(\boldsymbol{I}-\boldsymbol{H}_{\mathbf{0}}\right)^{-1} \boldsymbol{H}_{\mathbf{1}}=\underline{\pi}, \quad \underline{\pi} \mathbb{1}=1
$$


has a unique solution, and

$$
\begin{aligned}
& P_{\mathcal{X}(t)}\left(x_{0}, x_{1}, \ldots, x_{k}\right) \\
& =\underline{\pi} \boldsymbol{H}_{\mathbf{0}}{ }^{x_{0}-1} \boldsymbol{H}_{\mathbf{1}} \boldsymbol{H}_{\mathbf{0}}{ }^{x_{1}-1} \boldsymbol{H}_{\mathbf{1}} \ldots \boldsymbol{H}_{\mathbf{0}}{ }^{x_{k}-1} \boldsymbol{H}_{\mathbf{1}} \mathbb{1} .
\end{aligned}
$$

In this case we say that $\mathcal{X}(t)$ is a discrete rational arrival process with representation $\left(\boldsymbol{H}_{\mathbf{0}}, \boldsymbol{H}_{\mathbf{1}}\right)$ or, shortly, $\operatorname{DRAP}\left(\boldsymbol{H}_{\mathbf{0}}, \boldsymbol{H}_{\mathbf{1}}\right)$.

The size of the $\boldsymbol{H}_{0}$ and $\boldsymbol{H}_{1}$ matrices is also referred to as the order of the associated process. For brevity we will denote $n$-th order MGs, DPHs, DRAPs, etc. by $\operatorname{MG}(n), \operatorname{DPH}(n), \operatorname{DRAP}(n)$ etc., respectively.

An important consequence of Definition 3 is that $\boldsymbol{H}_{\mathbf{0}}$ and $\boldsymbol{H}_{1}$ have to be such that (6) is always non-negative.

Definition 4. If $\mathcal{X}(t)$ is a $\operatorname{DRAP}\left(\boldsymbol{H}_{\mathbf{0}}, \boldsymbol{H}_{\mathbf{1}}\right)$, where $\boldsymbol{H}_{\mathbf{0}}$ and $\boldsymbol{H}_{\mathbf{1}}$ are non-negative, we say that $\mathcal{X}(t)$ is a discrete Markov arrival process with representation $\left(\boldsymbol{H}_{\mathbf{0}}, \boldsymbol{H}_{\mathbf{1}}\right)$ or, shortly, DMAP $\left(\boldsymbol{H}_{\mathbf{0}}, \boldsymbol{H}_{\mathbf{1}}\right)$.

The matrix pairs satisfying the conditions of Definition 4 are called Markovian and the matrix pairs violating Definition 4 are called non-Markovian.

Definition 5. The correlation parameter $\gamma$ of a DRAP $\left(\boldsymbol{H}_{\mathbf{0}}, \boldsymbol{H}_{\mathbf{1}}\right)$ is the eigenvalue of $\left(\boldsymbol{I}-\boldsymbol{H}_{\mathbf{0}}\right)^{-1} \boldsymbol{H}_{\mathbf{1}}$ with the second largest absolute value.

One of the eigenvalues of $\left(\boldsymbol{I}-\boldsymbol{H}_{0}\right)^{-1} \boldsymbol{H}_{\mathbf{1}}$ is 1 , because $\left(\boldsymbol{H}_{\mathbf{0}}+\boldsymbol{H}_{\mathbf{1}}\right) \mathbb{1}=\mathbb{1}$, and the other eigenvalues are on the unit disk. If $\gamma$ is real, it is between -1 and 1. This parameter is especially important in the case of second-order DRAPs, as their $\rho_{k}$ lag- $k$ autocorrelation coefficient can be given as $\rho_{k}=\gamma^{k} c_{0}$, where $c_{0}$ depends only on the stationary inter-arrival time distribution of the process.

Similarly to DPHs, a DMAP has infinitely many different Markovian and non-Markovian representations (matrix pairs that satisfy (6)). One way to get a different representation of a $\operatorname{DMAP}\left(D_{0}, D_{1}\right)$ with the same size is the application of the similarity transformation

$$
H_{0}=T^{-1} D_{0} T, \quad H_{1}=T^{-1} D_{1} T,
$$

where $\boldsymbol{T}$ is an arbitrary non-singular matrix for which $T \mathbb{1}=\mathbb{1}$.

The (stationary) marginal distribution of the inter-event time of $\operatorname{DRAP}\left(\boldsymbol{H}_{\mathbf{0}}, \boldsymbol{H}_{\mathbf{1}}\right)$ is $\operatorname{MG}\left(\pi, \boldsymbol{H}_{\mathbf{0}}\right)$, where $\pi$ is the unique solution of (5). Similarly, the (stationary) marginal distribution of the inter-event time of $\operatorname{DMAP}\left(\boldsymbol{H}_{\mathbf{0}}, \boldsymbol{H}_{\mathbf{1}}\right)$ is $\operatorname{DPH}\left(\pi, \boldsymbol{H}_{\mathbf{0}}\right)$, where $\pi$ is the unique solution of (5).

2.3. Continuous phase type and matrix exponential distributions. The continuous counterparts of the above introduced models are defined as follows.
Definition 6. Let $\mathcal{X}$ be a continuous random variable with support on $\mathbb{R}^{+}$and cumulative distribution function (cdf)

$$
F_{X}(x)=\operatorname{Pr}(\mathcal{X}<x)=1-\alpha e^{A x} \mathbb{1},
$$

where $\alpha$ is a row vector of size $n, \boldsymbol{A}$ is a square matrix of size $n \times n$, and $\mathbb{1}$ is the column vector of ones of size $n$. In this case, we say that $\mathcal{X}$ is a matrix exponentially distributed with representation $(\alpha, \boldsymbol{A})$ or, shortly, $\operatorname{ME}(\alpha, \boldsymbol{A})$ distributed.

Definition 7. If $\mathcal{X}$ is an $\operatorname{ME}(\alpha, \boldsymbol{A})$ distributed random variable, where $\alpha$ and $\boldsymbol{A}$ have the following properties:

- $\alpha_{i} \geq 0, \alpha \mathbb{1}=1$ (there is no probability mass at $x=0$ ),

- $A_{i i}<0, A_{i j} \geq 0$ for $i \neq j, \boldsymbol{A} \mathbb{1} \leq 0$,

we say that $\mathcal{X}$ is phase type distributed with representation $(\alpha, \boldsymbol{A})$ or, shortly, $\mathrm{CPH}(\alpha, \boldsymbol{A})$ distributed.

The vector-matrix representations satisfying the conditions of Definition 7 are called Markovian.

The probability density function (pdf), the Laplace transform, and the moments of $\mathcal{X}$ are

$$
\begin{gathered}
f_{\mathcal{X}}(x)=-\alpha e^{\boldsymbol{A} x} \boldsymbol{A} \mathbb{1}, \\
\mu_{n}=E\left(\mathcal{X}^{n}\right)=n ! \alpha(-\boldsymbol{A})^{-n} \mathbb{1} .
\end{gathered}
$$

2.4. Continuous Markov arrival process and a continuous rational arrival process. Let $\mathcal{X}(t)$ be a point process on $\mathbb{R}^{+}$with a joint probability density function of inter-event times $f\left(x_{0}, x_{1}, \ldots, x_{k}\right)$ for $k=1,2, \ldots$.

Definition 8. $\mathcal{X}(t)$ is called a rational arrival process if there exists a finite $\left(\boldsymbol{H}_{\mathbf{0}}, \boldsymbol{H}_{\mathbf{1}}\right)$ square matrix pair such that $\left(\boldsymbol{H}_{\mathbf{0}}+\boldsymbol{H}_{\mathbf{1}}\right) \mathbb{1}=0$,

$$
\underline{\pi}\left(-\boldsymbol{H}_{\mathbf{0}}\right)^{-1} \boldsymbol{H}_{\mathbf{1}}=\underline{\pi}, \quad \underline{\pi} \mathbb{1}=1
$$

has a unique solution, and

$$
\begin{aligned}
& f\left(x_{0}, x_{1}, \ldots, x_{k}\right) \\
& \quad=\underline{\pi} e^{\boldsymbol{H}_{\mathbf{0}} x_{0}} \boldsymbol{H}_{\mathbf{1}} e^{\boldsymbol{H}_{\mathbf{0}} x_{1}} \boldsymbol{H}_{\mathbf{1}} \ldots e^{\boldsymbol{H}_{\mathbf{0}} x_{k}} \boldsymbol{H}_{\mathbf{1}} \mathbb{1} .
\end{aligned}
$$

In this case we say that $\mathcal{X}(t)$ is a rational arrival process with representation $\left(\boldsymbol{H}_{\mathbf{0}}, \boldsymbol{H}_{\mathbf{1}}\right)$ or, shortly, $\operatorname{RAP}\left(\boldsymbol{H}_{\mathbf{0}}, \boldsymbol{H}_{\mathbf{1}}\right)$.

Definition 9. If $\mathcal{X}(t)$ is a $\operatorname{RAP}\left(\boldsymbol{H}_{\mathbf{0}}, \boldsymbol{H}_{\mathbf{1}}\right)$, where $\boldsymbol{H}_{\mathbf{0}}$ and $\boldsymbol{H}_{1}$ have the following properties:

- $\boldsymbol{H}_{\mathbf{1}_{i j}} \geq 0$,

- $\boldsymbol{H}_{\mathbf{0}_{i i}}<0, \boldsymbol{H}_{\mathbf{0}_{i j}} \geq 0$ for $i \neq j, \boldsymbol{H}_{\mathbf{0}} \mathbb{1} \leq 0$,

we say that $\mathcal{X}(t)$ is a Markov arrival process with representation $\left(\boldsymbol{H}_{\mathbf{0}}, \boldsymbol{H}_{\mathbf{1}}\right)$ or, shortly, $\operatorname{MAP}\left(\boldsymbol{H}_{\mathbf{0}}, \boldsymbol{H}_{\mathbf{1}}\right)$.

Similarly to the discrete case, the representations satisfying the conditions of Definition 9 are called Markovian, and similarity transformations generate different representations of the same process. 


\section{Canonical form of second-order DPH distributions}

In this section we provide a canonical form for $\mathrm{DPH}(2)$ distributions. We start with characterizing the properties of all possible $\mathrm{MG}(2)$ distributions, i.e., distributions of the form (1), where $\boldsymbol{A}$ is a $2 \times 2$ matrix. Using this characterization we prove that all $\mathrm{MG}(2)$ distributions (thus all second-order DPH distributions) have a Markovian canonical form. After that we present the exact transformation method.

\subsection{Canonical form of $\operatorname{DPH}(2)$.}

Theorem 1. An MG(2) distribution has one of the following two forms:

- different eigenvalues:

$$
p_{i}=\operatorname{Pr}(\mathcal{X}=i)=a_{1} s_{1}^{i-1}+a_{2} s_{2}^{i-1},
$$

where $s_{1}, s_{2}$ are the eigenvalues of $\boldsymbol{A}$. These eigenvalues are real with $0<s_{1}<1, s_{1}>\left|s_{2}\right|$. Moreover, $a_{1}, a_{2}$ are such that

$$
\begin{gathered}
a_{1} \leq \frac{\left(1-s_{1}\right)\left(1-s_{2}\right)}{s_{1}-s_{2}}, \\
a_{2}=\left(1-s_{2}\right)\left(1-\frac{a_{1}}{1-s_{1}}\right) .
\end{gathered}
$$

Furthermore, $a_{1}>0$ if $s_{2} \geq 0$ and

$$
a_{1} \geq \frac{s_{2}\left(1-s_{1}\right)\left(1-s_{2}\right)}{s_{2}\left(1-s_{2}\right)-s_{1}\left(1-s_{1}\right)}
$$

if $s_{2}<0$;

- identical eigenvalues:

$$
p_{i}=\operatorname{Pr}(\mathcal{X}=i)=\left(a_{1}(i-1)+a_{2}\right) s^{i-1},
$$

where $s$ is the double eigenvalue of $\boldsymbol{A}$. This eigenvalue is real with $0<s<1$. Furthermore, $a_{1}, a_{2}$ are such that

$$
0<a_{1} \leq \frac{(1-s)^{2}}{s}, \quad a_{2}=\frac{(1-s)^{2}-a_{1} s}{1-s} .
$$

A vector matrix representation of the first form is

$$
\begin{aligned}
\alpha & =\left[\frac{a_{1}}{1-s_{1}}, \frac{a_{2}}{1-s_{2}}\right], \\
\boldsymbol{A} & =\left[\begin{array}{cc}
s_{1} & 0 \\
0 & s_{2}
\end{array}\right],
\end{aligned}
$$

and of the second form is

$$
\begin{aligned}
\alpha & =\left[\frac{a_{1}}{1-s}, \frac{a_{2}(1-s)-a_{1}(1-2 s)}{(1-s)^{2}}\right], \\
\boldsymbol{A} & =\left[\begin{array}{ll}
s & s \\
0 & s
\end{array}\right] .
\end{aligned}
$$

Proof. The first form covers the cases when the eigenvalues $s_{1}$ and $s_{2}$ of $\boldsymbol{A}$ are different and the second one when the eigenvalues are identical $\left(s_{1}=s_{2}=s\right)$. We discuss these cases separately.

Case 1: Different eigenvalues. First we show that the eigenvalues are real. Assume that $\boldsymbol{A}$ has a complex eigenvalue. In this case the other eigenvalue has to be its complex conjugate and $a_{1}$ and $a_{2}$ must be conjugates, too, to obtain real $p_{i}=a_{1} s_{1}^{i-1}+a_{2} s_{2}^{i-1}$ values. Let $\varphi$ be the argument of $a_{1}\left(a_{1}=\left|a_{1}\right| e^{i \varphi}\right)$, and $\psi$ the argument of $s_{1}$. Moreover, assume that $\psi \in(0, \pi)$. From $i=1$ we get that $\varphi \in[-\pi / 2, \pi / 2]$. Now consider the case $i=\lceil\pi / \psi\rceil+1$. The argument of $a_{1} s_{1}^{i-1}$ is $\varphi+(i-1) \psi$, and it is in $[\pi / 2,3 \pi / 2]$. This means that $p_{i}$ is negative since $a_{1} s_{1}^{i-1}$ and $a_{2} s_{2}^{i-1}$ are conjugates. Thus we get that the eigenvalues are real.

The two real eigenvalues have to be such that the one with the larger absolute value $\left(s_{1}\right)$ is positive, because it becomes dominant for large $i$ and $p_{i}$ would become negative for large $i$ with a negative dominant eigenvalue. Additionally, the dominant eigenvalue has to be less than one to ensure that the $p_{i}$ series has finite sum.

The relation of the $a_{1}, a_{2}$ coefficients is obtained from $\sum_{i} p_{i}=1$. The bound $a_{1}>0$ for the case $s_{2} \geq 0$ comes from the fact that $p_{i} \sim a_{1} s_{1}^{i-1}$ for large $i$, where $s_{1}$ is positive. A negative $a_{1}$ would result in negative $p_{i}$ for large $i$. If $s_{2}<0$, this is not enough, since $p_{i}$ can still be negative for smaller $i$ if $a_{2}$ is sufficiently large. In this case the lower bound for $a_{1}$ comes from $p_{2} \geq 0$, as

$$
\begin{aligned}
0 & \leq p_{2}=a_{1} s_{1}+a_{2} s_{2}, \\
0 & \leq a_{1} s_{1}+\left(1-s_{2}\right)\left(1-\frac{a_{1}}{1-s_{1}}\right) s_{2}, \\
0 & \leq a_{1} \frac{s_{2}\left(1-s_{2}\right)-s_{1}\left(1-s_{1}\right)}{1-s_{1}}+s_{2}\left(1-s_{2}\right), \\
a_{1} & \geq \frac{s_{2}\left(1-s_{1}\right)\left(1-s_{2}\right)}{s_{1}\left(1-s_{1}\right)-s_{2}\left(1-s_{2}\right)} .
\end{aligned}
$$

The upper bound of $a_{1}$ can be derived from $p_{1} \geq 0$, since

$$
\begin{aligned}
0 & \leq p_{1}=a_{1}+a_{2}, \\
0 & \leq a_{1}+\left(1-s_{2}\right)\left(1-\frac{a_{1}}{1-s_{1}}\right), \\
0 & \leq a_{1} \frac{s_{2}-s_{1}}{1-s_{1}}+\left(1-s_{2}\right), \\
a_{1} & \leq \frac{\left(1-s_{1}\right)\left(1-s_{2}\right)}{s_{1}-s_{2}}
\end{aligned}
$$

Case 2: Identical eigenvalues. First we show that the eigenvalue is real and non-negative. If $s$ is complex or negative in (14), then $p_{i} \sim a_{1}(i-1) s^{i-1}$ for large $i$, which becomes complex or negative, respectively, for any $a_{1}$ in the case of two consecutive large values of $i$. 
The inequality $s<1$ comes from the fact that the series $p_{i}$ has a finite sum.

Similarly to the previous case, the relation of the $a_{1}$, $a_{2}$ coefficients is obtained from $\sum_{i} p_{i}=1$ and the bound $a_{1}>0$ comes from the fact that $p_{i} \sim a_{1}(i-1) s^{i-1}$ for large $i$, where $s$ is positive. A negative $a_{1}$ would result in negative $p_{i}$ for large $i$. The upper bound of $a_{1}$ comes from $p_{1} \geq 0$, since

$$
\begin{aligned}
0 & \leq p_{1}=a_{2}, \\
0 & \leq \frac{(1-s)^{2}-a_{1} s}{1-s}, \\
a_{1} & \leq \frac{(1-s)^{2}}{s} .
\end{aligned}
$$

Theorem 2. If $\mathcal{X}$ is $\mathrm{MG}(2)$ distributed with two distinct positive eigenvalues $\left(0<s_{2}<s_{1}<1\right)$, then it can be represented as $\operatorname{ADPH}(\alpha, \boldsymbol{A})$, where

$$
\begin{aligned}
\alpha & =\left[\frac{a_{1}\left(s_{1}-s_{2}\right)}{\left(1-s_{1}\right)\left(1-s_{2}\right)}, \frac{a_{1}+a_{2}}{1-s_{2}}\right], \\
\boldsymbol{A} & =\left[\begin{array}{cc}
s_{1} & 1-s_{1} \\
0 & s_{2}
\end{array}\right] .
\end{aligned}
$$

Proof. The $(\alpha, \boldsymbol{A})$ vector-matrix pair is such that $p_{i}=$ $\alpha \boldsymbol{A}^{i-1}(\mathbb{1}-\boldsymbol{A} \mathbb{1})=a_{1} s_{1}^{i-1}+a_{2} s_{2}^{i-1}$. Matrix $\boldsymbol{A}$ obviously satisfies the conditions of Definition 2 when $0<s_{2}<$ $s_{1}<1$. It remains to show that $\alpha$ is non-negative when $0<s_{2}<s_{1}<1,0<a_{1}$, and $p_{1} \geq 0$. In the first element of $\alpha$ we have $a_{1}>0, s_{1}-s_{2}>0, s_{1}-1<0, s_{2}-1<0$, from which it is positive. In the second element we have $a_{1}+a_{2}=p_{1} \geq 0$ and $1-s_{2}>0$. Note that $\alpha \mathbb{1}=1$ when

$$
a_{2}=\left(1-s_{2}\right)\left(1-\frac{a_{1}}{1-s_{1}}\right) \text {. }
$$

Theorem 3. If $\mathcal{X}$ is $\mathrm{MG}(2)$ distributed with a dominant positive and a negative eigenvalue $\left(s_{2}<0<s_{1}<1\right.$ and $\left.s_{1}+s_{2}>0\right)$, then it can be represented as $\operatorname{DPH}(\alpha, \boldsymbol{A})$, where

$$
\begin{gathered}
\alpha=\left[\frac{a_{1} s_{1}+a_{2} s_{2}}{\left(1-s_{1}\right)\left(1-s_{2}\right)}, \frac{\left(a_{1}+a_{2}\right)\left(1-s_{1}-s_{2}\right)}{\left(1-s_{1}\right)\left(1-s_{2}\right)}\right], \\
\boldsymbol{A}=\left[\begin{array}{cc}
1-\beta_{1} & \beta_{1} \\
\beta_{2} & 0
\end{array}\right], \\
\beta_{1}=1-s_{1}-s_{2}, \quad \beta_{2}=\frac{s_{1} s_{2}}{s_{1}+s_{2}-1} .
\end{gathered}
$$

Proof. The eigenvalues of $\boldsymbol{A}$ are $s_{1}, s_{2}$ and the $(\alpha, \boldsymbol{A})$ pair is such that $p_{i}=\alpha \boldsymbol{A}^{i-1}(\mathbb{1}-\boldsymbol{A} \mathbb{1})=a_{1} s_{1}^{i-1}+a_{2} s_{2}^{i-1}$.
Parameters $\beta_{1}$ and $\beta_{2}$ are positive and less than 1 from which matrix $\boldsymbol{A}$ satisfies the conditions of Definition 2]

It remains to show that $\alpha$ is non-negative when $s_{2}<$ $0<s_{1}<1,1>s_{1}>s_{1}+s_{2}>0$ and $p_{1}, p_{2} \geq 0$. For the first element of $\alpha$, we have $a_{1} s_{1}+a_{2} s_{2}=p_{2} \geq 0$, $s_{1}-1<0, s_{2}-1<0$, from which it is positive, and for the numerator of the second element we have $a_{1}+a_{2}=$ $p_{1} \geq 0$ and $1-s_{1}-s_{2}>0$. The denominator of the second element is the same as that of the first one. Thus the second element of $\alpha$ is also non-negative.

Theorem 4. If $\mathcal{X}$ is $\mathrm{MG}(2)$ distributed with two identical eigenvalues $\left(0<s=s_{2}=s_{1}<1\right)$, then it can be represented as $\operatorname{ADPH}(\alpha, \boldsymbol{A})$, where

$$
\alpha=\left[\frac{a_{1} s}{(1-s)^{2}}, \frac{a_{2}}{1-s}\right], \quad \boldsymbol{A}=\left[\begin{array}{cc}
s & 1-s \\
0 & s
\end{array}\right] .
$$

Proof. The $(\alpha, \boldsymbol{A})$ vector-matrix pair is such that $p_{i}=$ $\alpha \boldsymbol{A}^{i-1}(\mathbb{1}-\boldsymbol{A} \mathbb{1})=\left(a_{1}(i-1)+a_{2}\right) s^{i-1}$, and matrix $\boldsymbol{A}$ satisfies the conditions of Definition 2 when $0<s<1$.

It remains to show that $\alpha$ is non-negative when $0<$ $s<1,0<a_{1}$ and $p_{1} \geq 0$. All terms of the elements of $\alpha$ are non-negative since $a_{2}=p_{1} \geq 0$.

Theorems 2 have the following consequences.

Corollary 1. The vector-matrix representations in Theorems 24 can be used as canonical representations of $\mathrm{DPH}(2)$ and $\mathrm{MG}(2)$ distributions.

Corollary 2. We have

$$
\text { second-order DPH } \equiv \text { second-order } M G \text {. }
$$

Moreover,

$$
\text { second-order } A D P H \equiv \text { second-order } M G
$$

with positive eigenvalues.

Corollary 3. If the eigenvalues of second-order $\mathrm{MG}(\gamma, \boldsymbol{G})$ are positive and its canonical representation is $\operatorname{ADPH}(\alpha, \boldsymbol{A})$, then $\mathrm{ME}(\gamma, \boldsymbol{G}-\boldsymbol{I})$ is a matrix exponential distribution whose canonical $\mathrm{ACPH}$ representation (Cumani's canonical form) is $\mathrm{ACPH}(\alpha, \boldsymbol{A}-\boldsymbol{I})$.

Proof. The matrix of the canonical representation $\operatorname{ADPH}(\alpha, \boldsymbol{A})$ has the form

$$
\left[\begin{array}{cc}
s_{1} & 1-s_{1} \\
0 & s_{2}
\end{array}\right]
$$

where $1>s_{1} \geq s_{2}>0$. Consequently, $\boldsymbol{A}-\boldsymbol{I}$ is a matrix of an ACPH distribution in Cumani's canonical form with eigenvalues $0>s_{1}-1 \geq s_{2}-1>-1$.

Furthermore, since $\operatorname{ME}(\gamma, \boldsymbol{G}-\boldsymbol{I})$ and $\operatorname{ACPH}(\alpha, \boldsymbol{A}-$ $\boldsymbol{I})$ represent the same distribution, $\operatorname{ME}(\gamma, \boldsymbol{G}-\boldsymbol{I})$ is a valid ME distribution. 
3.2. Transformation of $\operatorname{DPH}(2)$ to the canonical form. The introduced canonical representations can be obtained from a general vector-matrix representation with the following similarity transformation.

Corollary 4. If the eigenvalues of a second-order $\operatorname{MG}(\gamma, \boldsymbol{G})$ are $0<s_{2}<s_{1}<1$, then its canonical representation is $\operatorname{ADPH}\left(\alpha=\gamma \boldsymbol{B}, \boldsymbol{A}=\boldsymbol{B}^{-1} \boldsymbol{G} \boldsymbol{B}\right)$, where matrix $\boldsymbol{B}$ is composed of column vectors

$$
b_{1}=\mathbb{1}-b_{2}
$$

and

$$
b_{2}=\frac{1}{1-s_{2}}(\mathbb{1}-\boldsymbol{G} \mathbb{1}) .
$$

Proof. Matrix $\boldsymbol{B}$ is obtained as the solution of $\boldsymbol{B} \mathbb{1}=$ $b_{1}+b_{2}=\mathbb{1}$ and

$$
\boldsymbol{G} \boldsymbol{B}=\boldsymbol{B}\left[\begin{array}{cc}
s_{1} & 1-s_{1} \\
0 & s_{2}
\end{array}\right],
$$

whose column vector form is $\boldsymbol{G} b_{1}=s_{1} b_{1}$ and $\boldsymbol{G} b_{2}=$ $\left(1-s_{1}\right) b_{1}+s_{2} b_{2}$. Consequently,

$$
\boldsymbol{A}=\left[\begin{array}{cc}
s_{1} & 1-s_{1} \\
0 & s_{2}
\end{array}\right]
$$

The proofs for the subsequent corollaries in this section follow the same pattern and are omitted.

Corollary 5. If the eigenvalues of a second-order $\mathrm{MG}(\gamma, \boldsymbol{G})$ are $s_{2}<0<s_{1}<1$, then its canonical representation is $\mathrm{ADPH}$

$$
\left(\gamma \boldsymbol{B},\left[\begin{array}{cc}
s_{1}+s_{2} & 1-s_{1}-s_{2} \\
\frac{s_{1} s_{2}}{s_{1}+s_{2}-1} & 0
\end{array}\right]\right),
$$

where matrix $\boldsymbol{B}$ is composed of column vectors

$$
b_{1}=\mathbb{1}-b_{2}
$$

and

$$
b_{2}=\frac{1-s_{1}-s_{2}}{\left(1-s_{1}\right)\left(1-s_{2}\right)}(\mathbb{1}-\boldsymbol{G} \mathbb{1}) .
$$

Corollary 6. If the eigenvalues of a second-order $\operatorname{MG}(\gamma, \boldsymbol{G})$ are $s=s_{1}=s_{2}<1$, then its canonical representation is

$$
\operatorname{ADPH}\left(\gamma \boldsymbol{B},\left[\begin{array}{cc}
s & 1-s \\
0 & s
\end{array}\right]\right),
$$

where matrix $\boldsymbol{B}$ is composed of column vectors $b_{1}=\mathbb{1}-$ $b_{2}$ and $b_{2}=\frac{1}{1-s}(\mathbb{1}-\boldsymbol{G} \mathbb{1})$.
The presented similarity transformations can be used as transformation methods to compute the canonical representation from a general (Markovian or non-Markovian) vector matrix representation. As an example, a simple implementation of Corollary 4 is presented as Algorithm 1 .

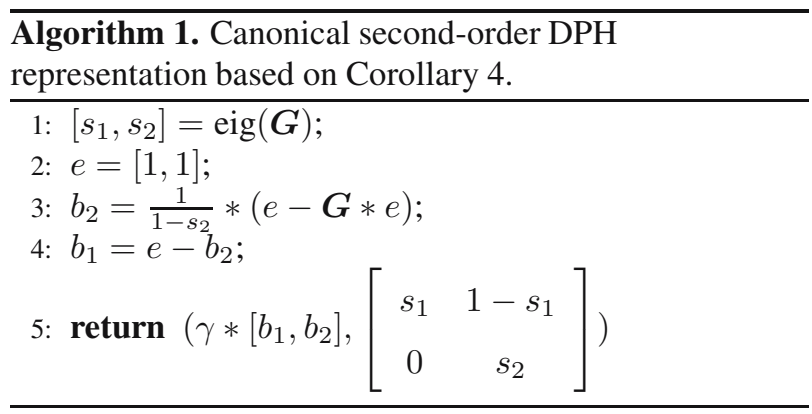

\section{Canonical form of third-order DPH distributions}

In the previous section we proved that the whole MG(2) class can be represented with Markovian vector-matrix pairs. That is why we started with the characterization of the second-order MG class. For third-order distributions the same does not hold, that is, $\operatorname{DPH}(3) \not \equiv \operatorname{MG}(3)$. Due to this difference we follow a different approach here and show only that a transformation with a given similarity matrix results in a Markovian canonical form for all $\mathrm{DPH}(3) \mathrm{s}$.

Similarly to the second-order case, the canonical representations of $\mathrm{DPH}(3)$ distributions are classified according to the eigenvalue structure of the distribution. We encode the eigenvalues in decreasing absolute values and denote by $\mathrm{N}$ the ones with negative real parts and by $\mathrm{P}$ the ones with non-negative real parts. For example PNP, means that $1 \geq\left|s_{1}\right| \geq\left|s_{2}\right| \geq\left|s_{3}\right|$ and $\operatorname{Re}\left(s_{1}\right) \geq$ $\operatorname{Re}\left(s_{3}\right) \geq 0>\operatorname{Re}\left(s_{2}\right)$, where $s_{i}, i=1,2,3$ denote the eigenvalues. Since the eigenvalue with the largest absolute value (dominant eigenvalue) has to be real and positive (to ensure positive probabilities in (1) for large $i$ ), we have the following cases: PPP, PPN, PNP, PNN. Complex (conjugate) eigenvalues can occur only in the case of PPP and PNN.

4.1. Case PPP. Following the pattern of Corollary 3 we define the canonical form in the PPP case based on the canonical representation of $\mathrm{CPH}(3)$ distributions.

Theorem 5. If the eigenvalues of a third-order $\operatorname{DPH}(\gamma, \boldsymbol{G})$ are all non-negative, we define the canonical form as follows. The vector-matrix pair $(\gamma, \boldsymbol{G}-\boldsymbol{I})$ defines a $\mathrm{CPH}(3)$. Let $(\alpha, \boldsymbol{A})$ be the canonical representation of $\operatorname{CPH}(\gamma, \boldsymbol{G}-\boldsymbol{I})$ as defined by Horváth and Telek (2009). The canonical representation of $\mathrm{DPH}(\gamma, \boldsymbol{G})$ is $(\alpha, \boldsymbol{A}+\boldsymbol{I})$. 
Proof. The complete proof of the theorem requires the introduction of the procedure defined by Horváth and Telek (2009). Here we only demonstrate the result for the case when the canonical representation of $\mathrm{CPH}(\gamma, \boldsymbol{G}-\boldsymbol{I})$ is acyclic. When the eigenvalues of $\boldsymbol{G}$ are $1>s_{1} \geq s_{2} \geq$ $s_{3}>0$, the eigenvalues of $\boldsymbol{G}-\boldsymbol{I}$ are $0>s_{1}-1 \geq$ $s_{2}-1 \geq s_{3}-1>-1$. In this case the matrix of the acyclic canonical form of $\mathrm{CPH}(\gamma, \boldsymbol{G}-\boldsymbol{I})$ is

$$
\boldsymbol{A}=\left[\begin{array}{ccc}
s_{3}-1 & 0 & s^{*}=0 \\
1-s_{2} & s_{2}-1 & 0 \\
0 & 1-s_{1} & s_{1}-1
\end{array}\right]
$$

and the associated vector $\alpha$ is non-negative. Finally, $\boldsymbol{A}+\boldsymbol{I}$ is non-negative and the associated exit probability vector,

$$
\mathbb{1}-\boldsymbol{A} \mathbb{1}=\left[1-s_{3}, 0,0\right]^{T},
$$

is non-negative as well.

In the general case, $s^{*}$ might be positive and $s_{i}-1$, $i=1,2,3$ are not the eigenvalues of $\boldsymbol{A}$, but also in that case the elements of $\boldsymbol{A}+\boldsymbol{I}$ and $\mathbb{1}-\boldsymbol{A} \mathbb{1}$ are non-negative.

The rest of the cases require the introduction of new canonical structures.

\subsection{Case PPN.}

Theorem 6. If the eigenvalues of a third-order $\operatorname{DPH}(\gamma, \boldsymbol{G})$ satisfy $1>\left|s_{1}\right| \geq\left|s_{2}\right| \geq\left|s_{3}\right|$ and $\operatorname{Re}\left(s_{1}\right) \geq$ $\operatorname{Re}\left(s_{2}\right)>0>\operatorname{Re}\left(s_{3}\right)$, then its canonical representation is $\operatorname{DPH}(\gamma \boldsymbol{B}, \boldsymbol{A})$, where

$$
\begin{gathered}
\boldsymbol{A}=\left[\begin{array}{ccc}
x_{1} & 1-x_{1} & 0 \\
0 & x_{2} & 1-x_{2} \\
0 & x_{3} & 0
\end{array}\right], \\
x_{1}=s_{1}, \quad x_{2}=s_{2}+s_{3}, \quad x_{3}=\frac{-s_{2} s_{3}}{1-s_{2}-s_{3}},
\end{gathered}
$$

and matrix $\boldsymbol{B}$ is composed of column vectors

$$
\begin{gathered}
b_{1}=\mathbb{1}-b_{2}-b_{3}, \\
b_{2}=\frac{1}{\left(1-x_{2}\right)\left(1-x_{3}\right)} \boldsymbol{G}(\mathbb{1}-\boldsymbol{G} \mathbb{1}), \\
b_{3}=\frac{1}{1-x_{3}}(\mathbb{1}-\boldsymbol{G} \mathbb{1}) .
\end{gathered}
$$

Proof. The eigenvalues of the canonical matrix are $s_{1}, s_{2}, s_{3}$. We need to prove that $0 \leq x_{i}<1$ and $\gamma b_{i} \geq 0$ for $i=1,2,3$. Based on the eigenvalue conditions of the
PPN case, the validity of $x_{1}$ and $x_{2}$ is immediate. For $x_{3}$ it is easy to see that $x_{3}>0$. For the other limit we have

$$
\begin{aligned}
\frac{-s_{2} s_{3}}{1-s_{2}-s_{3}} & <1, \\
0 & <1-s_{2}-s_{3}+s_{2} s_{3}, \\
0 & <\underbrace{\left(1-s_{2}\right)}_{>0} \underbrace{\left(1-s_{3}\right)}_{>0} .
\end{aligned}
$$

The elements of $b_{2}$ and $b_{3}$ are non-negative, because $(\mathbb{1}-G \mathbb{1})$ and $G(\mathbb{1}-G \mathbb{1})$ are the one-and two-step exit probability vectors of $\operatorname{DPH}(\gamma, \boldsymbol{G})$ and $0 \leq x_{2}, x_{3}<1$.

All that is left is to prove that $b_{1}$ is non-negative. By substituting into $b_{1}=\mathbb{1}-\left(b_{2}+b_{3}\right)$, we get

$$
b_{2}+b_{3}=\left(\frac{1}{1-x_{2}} \boldsymbol{G}+\boldsymbol{I}\right) \frac{1}{1-x_{3}}(\boldsymbol{I}-\boldsymbol{G}) \mathbb{1}=\boldsymbol{M} \mathbb{1},
$$

which is the product of a matrix (denoted by $M$ above) and the vector $\mathbb{1}$. Let us examine $\sigma_{i}, i=1,2,3$ the eigenvalues of $M$. The matrix $M$ is a polynomial function of $\boldsymbol{G}$. Therefore, its eigenvalues can be calculated using (25) as

$$
\sigma_{i}=\left(\frac{1}{1-x_{2}} s_{i}+1\right) \frac{1}{1-x_{3}}\left(1-s_{i}\right) \mathbb{1} .
$$

First note that $\sigma_{i} \geq 0, i=1,2,3$, as $x_{2}$ and $x_{3}$ are less than 1 . Substituting into $x_{2}$ and $x_{3}$ for $i=1$, we get

$$
\begin{aligned}
\sigma_{1} & =\left(\frac{1}{1-x_{2}} s_{1}+1\right) \frac{1}{1-x_{3}}\left(1-s_{1}\right) \\
& =\left(\frac{1}{1-s_{2}-s_{3}} s_{1}+1\right) \frac{1-s_{2}-s_{3}}{\left(1-s_{2}\right)\left(1-s_{3}\right)}\left(1-s_{1}\right) \\
& =\left(s_{1}+1-s_{2}-s_{3}\right) \frac{1}{\left(1-s_{2}\right)\left(1-s_{3}\right)}\left(1-s_{1}\right) \\
& =\frac{1-s_{1}}{1-s_{2}}\left(1+\frac{s_{1}-s_{2}}{1-s_{3}}\right) \leq \frac{1-s_{1}}{1-s_{2}}\left(1+s_{1}-s_{2}\right),
\end{aligned}
$$

which is less than 1 as

$$
\begin{aligned}
\frac{1-s_{1}}{1-s_{2}}\left(1+s_{1}-s_{2}\right) & \leq 1 \\
\left(1-s_{1}\right)\left(1+s_{1}-s_{2}\right) & \leq\left(1-s_{2}\right), \\
-s_{1}^{2}+s_{1} s_{2} & \leq 0 .
\end{aligned}
$$

For $i=2$,

$$
\begin{aligned}
\sigma_{i} & =\left(\frac{1}{1-x_{2}} s_{i}+1\right) \frac{1}{1-x_{3}}\left(1-s_{i}\right) \\
& =\left(\frac{1}{1-s_{2}-s_{3}} s_{2}+1\right) \frac{1-s_{2}-s_{3}}{\left(1-s_{2}\right)\left(1-s_{3}\right)}\left(1-s_{2}\right) \\
& =\left(s_{2}+1-s_{2}-s_{3}\right) \frac{1}{\left(1-s_{2}\right)\left(1-s_{3}\right)}\left(1-s_{2}\right)=1 .
\end{aligned}
$$


In much the same way, $\sigma_{3}=1$ can be derived. Thus the eigenvalues of $M$ are between 0 and 1. This means that the transformation $M \mathbb{1}$ cannot increase the length of $\mathbb{1}$, i.e., the smallest element of $b_{2}+b_{3}=M \mathbb{1}$ is less than 1. In other words, at least one of the elements of $b_{1}=\mathbb{1}-\left(b_{2}+b_{3}\right)$ is positive. However, from the first column of the matrix equation $\boldsymbol{G} \boldsymbol{B}=\boldsymbol{B} \boldsymbol{A}$ we have another expression for $b_{1}, x_{1} b_{1}=\boldsymbol{G} b_{1}$. That is, $x_{1}=s_{1}$ is the largest eigenvalue of $\boldsymbol{G}$, and $b_{1}$ is the associated eigenvector, which is either strictly positive or strictly negative according to the Perron-Frobenius theorem. Consequently, $b_{1}$ is strictly positive. The elements of $\gamma$ are non-negative and therefore $\gamma b_{i}, i=$ $1,2,3$ are non-negative as well. This completes the proof.

4.3. Case PNP. The PNP case exhibits the widest set of representations. Here the eigenvalues are real and such that $0<s_{3}<-s_{2}<s_{1}<1$. Let the eigenvalue representation of the distribution be $p_{i}=\gamma \boldsymbol{G}^{i-1}(\mathbb{1}-$ $\boldsymbol{G} \mathbb{1})=\sigma_{1} s_{1}^{i-1}+\sigma_{2} s_{2}^{i-1}+\sigma_{3} s_{3}^{i-1}$. Using this notation, we first define the required representations.

Definition 10. The PNP representation of the distribution is

$$
\alpha=\gamma \boldsymbol{B}, \quad \boldsymbol{A}=\left[\begin{array}{ccc}
x_{1} & 1-x_{1} & 0 \\
x_{2} & 0 & 1-x_{2} \\
0 & x_{3} & 0
\end{array}\right]
$$

where

$$
\begin{gathered}
x_{1}=-a_{2}, \\
x_{2}=\frac{a_{0}-a_{1} a_{2}}{a_{2}\left(1+a_{2}\right)}, \\
x_{3}=\frac{a_{0}\left(1+a_{2}\right)}{a_{0}-a_{2}-a_{1} a_{2}-a_{2}^{2}},
\end{gathered}
$$

$a_{0}, a_{1}$, and $a_{2}$ are the coefficients of the characteristic polynomial of $G$, i.e.,

$$
\begin{gathered}
a_{0}=-s_{1} s_{2} s_{3}, \\
a_{1}=s_{1} s_{2}+s_{1} s_{3}+s_{2} s_{3}, \\
a_{2}=-s_{1}-s_{2}-s_{3} .
\end{gathered}
$$

The matrix $\boldsymbol{B}$ is composed of column vectors

$$
\begin{gathered}
b_{1}=\mathbb{1}-b_{2}-b_{3}, \\
b_{2}=\frac{1}{\left(1-x_{2}\right)\left(1-x_{3}\right)} \boldsymbol{G}(\mathbb{1}-\boldsymbol{G} \mathbb{1}), \\
b_{3}=\frac{1}{1-x_{3}}(\mathbb{1}-\boldsymbol{G} \mathbb{1}) .
\end{gathered}
$$

Definition 11. The $P N P+$ representation of the distribution is

$$
\begin{gathered}
\alpha=\left[\frac{\sigma_{3}}{1-s_{3}}, \frac{\sigma_{1} s_{1}+\sigma_{2} s_{2}}{\left(1-s_{1}\right)\left(1-s_{2}\right)}, \frac{\left(\sigma_{1}+\sigma_{2}\right)\left(1-s_{1}-s_{2}\right)}{\left(1-s_{1}\right)\left(1-s_{2}\right)}\right], \\
\boldsymbol{A}=\left[\begin{array}{ccc}
x_{1} & 0 & 0 \\
0 & x_{2} & 1-x_{2} \\
0 & x_{3} & 0
\end{array}\right], \\
x_{1}=s_{3}, \quad x_{2}=s_{1}+s_{2}, \quad x_{3}=\frac{-s_{1} s_{2}}{1-s_{1}-s_{2}} .
\end{gathered}
$$

Definition 12. The $P N P++$ representation of the distribution is

$$
\begin{gathered}
\alpha=\left[\frac{\sigma_{1}+\sigma_{2}+\sigma_{3}}{1-s_{3}}, \frac{\sigma_{1} s_{1}\left(s_{1}-s_{3}\right)+\sigma_{2} s_{2}\left(s_{2}-s_{3}\right)}{\left(1-s_{1}\right)\left(1-s_{2}\right)\left(1-s_{3}\right)},\right. \\
\left.\frac{\left(1-s_{1}-s_{2}\right)\left(\sigma_{1} s_{1}+\sigma_{2} s_{2}-\left(\sigma_{1}+\sigma_{2}\right) s_{3}\right)}{\left(1-s_{1}\right)\left(1-s_{2}\right)\left(1-s_{3}\right)}\right], \\
\boldsymbol{A}=\left[\begin{array}{ccc}
x_{1} & 0 & 0 \\
0 & x_{2} & 1-x_{2} \\
1-x_{3} & x_{3} & 0
\end{array}\right], \\
x_{1}=s_{3}, \quad x_{2}=s_{1}+s_{2}, \quad x_{3}=\frac{-s_{1} s_{2}}{1-s_{1}-s_{2}} .
\end{gathered}
$$

Theorem 7. When the eigenvalues are such that $0<$ $s_{3}<-s_{2}<s_{1}<1$, the generator matrices of the representations PNP, PNP+ and PNP are Markovian.

Proof. (PNP representation) Let $\lambda_{i}=-s_{i}$ for $i=1,2,3$. In this case, $\lambda_{2}$ is strictly positive and so $\lambda_{1}$ is strictly negative, while $\lambda_{3}$ is non-positive. Consequently, $a_{0}=$ $\lambda_{1} \lambda_{2} \lambda_{3} \geq 0$. The positivity of $x_{1}=-a_{2}$ follows from the fact that the sum of the eigenvalues of $\boldsymbol{G}$ is positive:

$$
\begin{aligned}
1+a_{2}=\underbrace{1+\lambda_{1}}_{>0}+\underbrace{\lambda_{2}+\lambda_{3}}_{\geq 0} & >0, \\
1 & >-a_{2}, \\
1 & >x_{1} .
\end{aligned}
$$

The first inequality follows from $-1<\lambda_{1}$ and $\left|\lambda_{3}\right| \leq\left|\lambda_{2}\right|$. The next inequality also follows from $-1<\lambda_{1}, \lambda_{3}$ and $0<\lambda_{2}$ :

$$
1+a_{0}+a_{1}+a_{2}=\left(1+\lambda_{1}\right)\left(1+\lambda_{2}\right)\left(1+\lambda_{3}\right)>0 .
$$

In the following, we have that $-a_{2}<1$. From that we get $a_{0} \geq-a_{2} a_{0}$.

The denominator of $x_{3}$ is

$$
\begin{aligned}
& a_{0}-a_{2}-a_{1} a_{2}-a_{2}^{2} \geq \\
& -\underbrace{a_{2}}_{<0}(\underbrace{1+a_{1}+a_{2}+a_{0}}_{>0})>0 .
\end{aligned}
$$


In the numerator of $x_{3} a_{0}$ is non-negative and $1+a_{2}$ is positive. Therefore, $x_{3}$ is non-negative. We need to show that $x_{3}<1$ :

$$
\begin{aligned}
x_{3} & <1, \\
a_{0}+a_{0} a_{2} & <a_{0}-a_{2}-a_{1} a_{2}-a_{2}^{2}, \\
0 & <-a_{2}\left(1+a_{0}+a_{1}+a_{2}\right),
\end{aligned}
$$

which was proven in (36). Finally, let us consider $x_{2}$ :

$$
\begin{aligned}
x_{2} & <1, \\
a_{0}-a_{1} a_{2} & >a_{2}\left(1+a_{2}\right), \\
a_{0}-a_{2}-a_{1} a_{2}-a_{2}^{2} & >0 .
\end{aligned}
$$

We have here that the eigenvalues of $\lambda_{i}$ are decreasing and only $\lambda_{2}$ is positive:

$$
x_{2}=\frac{-(\overbrace{\lambda_{1}+\lambda_{2}}^{\leq 0})(\overbrace{\lambda_{1}+\lambda_{3}}^{\leq 0})(\overbrace{\lambda_{2}+\lambda_{3}}^{\geq \underbrace{x_{1}}_{>0}})}{\underbrace{\left(1-x_{1}\right.}_{>0})} \geq 0 .
$$

$P N P+$ and $P N P++$ representations. In these cases the properties of $x_{i}$ are easy to read from the eigenvalue conditions and we have that $0<x_{1}, x_{2}, x_{3}<1$.

Conjecture 1. One of the PNP, the PNP+ and the PNP++ representations of a $\mathrm{DPH}(3)$ with $P N P$ eigenvalues is Markovian.

Proof. We could analytically treat several special cases of the DPH(3) PNP class, but we do not have a formal proof which covers the whole class. Apart from the analytical treatment of the special cases, we also completed an exhaustive numerical investigation and have not found any counterexample yet.

According to our numerical investigations, the $\mathrm{PNP}++$ representation covers (transforms to a Markovian representation) the largest set of randomly generated $\mathrm{DPH}(3) \mathrm{s}$. The second one is the PNP representation, and the PNP+ representation covers the least among our randomly generated DPH(3)s. Among $400000 \mathrm{DPH}(3) \mathrm{s}$ with PNP eigenvalues, there are $\sim 300$ ones whose PNP++ and PNP representations are non-Markovian and whose $\mathrm{PNP}+$ representation is Markovian.

\subsection{Case PNN.}

Theorem 8. If the eigenvalues of a third-order $\operatorname{DPH}(\gamma, \boldsymbol{G})$ are $1>\left|s_{1}\right| \geq\left|s_{2}\right| \geq\left|s_{3}\right|, \operatorname{Re}\left(s_{1}\right)>0>$ $\operatorname{Re}\left(s_{3}\right) \geq \operatorname{Re}\left(s_{2}\right)$ and $\left|s_{2}\right|^{2} \leq 2 s_{1}\left(-\operatorname{Re}\left(s_{2}\right)\right)$, then its canonical representation is $\operatorname{DPH}(\gamma \boldsymbol{B}, \boldsymbol{A})$, where

$$
\boldsymbol{A}=\left[\begin{array}{ccc}
x_{1} & 1-x_{1} & 0 \\
x_{2} & 0 & 1-x_{2} \\
x_{3} & 0 & 0
\end{array}\right],
$$

$$
x_{1}=-a_{2}, \quad x_{2}=\frac{-a_{1}}{1+a_{2}}, \quad x_{3}=\frac{-a_{0}}{1+a_{1}+a_{2}},
$$

the matrix elements are defined based on the coefficients of the characteristic polynomial of $\boldsymbol{G}$,

$$
\begin{gathered}
a_{0}=-s_{1} s_{2} s_{3}, \quad a_{1}=s_{1} s_{2}+s_{1} s_{3}+s_{2} s_{3}, \\
a_{2}=-s_{1}-s_{2}-s_{3},
\end{gathered}
$$

and the matrix $\boldsymbol{B}$ is composed of column vectors

$$
\begin{gathered}
b_{1}=\mathbb{1}-b_{2}-b_{3}, \quad b_{2}=\frac{1}{\left(1-x_{2}\right)\left(1-x_{3}\right)} \boldsymbol{G}(\mathbb{1}-\boldsymbol{G} \mathbb{1}), \\
b_{3}=\frac{1}{1-x_{3}}(\mathbb{1}-\boldsymbol{G} \mathbb{1}) .
\end{gathered}
$$

Proof. The eigenvalues of the canonical matrix are $s_{1}, s_{2}, s_{3}$. We need to prove that $0 \leq x_{i}<1$ and $\gamma b_{i} \geq 0$ for $i=1,2,3$.

Let $\lambda_{i}=-s_{i}$ for $i=1,2,3$. The statements about $a_{2}$ in the PNP case are also valid for this case. The trace of matrix $G$ (the sum of its diagonal elements) equals the sum of its eigenvalues, and so the sum of the eigenvalues, as well as $-a_{2}$, are non-negative. Consequently, $0 \leq$ $x_{1}<1$. Now we consider $x_{2}$. Since $\left(1+a_{2}\right)$ is positive, we need to show that $a_{1}$ is non-positive.

If the eigenvalues are all real, then we can write

$$
a_{1}=\underbrace{s_{1} s_{2}}_{<0}+\underbrace{s_{3}}_{<0} \underbrace{\left(s_{1}+s_{2}\right)}_{\geq 0},
$$

which is the sum of a negative and a non-positive number. Accordingly, the result will be negative as well.

If $s_{2}$ and $s_{3}$ are complex conjugates, we can write them as $s_{2}=-u+\mathfrak{i} v$ and $s_{3}=-u-\mathfrak{i} v$, where $u, v$ are positive reals. With this notation,

$$
\begin{aligned}
a_{1} & =s_{1}(-u+\mathfrak{i} v)+s_{1}(-u-\mathfrak{i} v)+\left(u^{2}+v^{2}\right) \\
& =u^{2}+v^{2}-2 s_{1} u \leq 0,
\end{aligned}
$$

where the last inequality comes from $\left|s_{2}\right|^{2} \leq$ $2 s_{1}\left(-\operatorname{Re}\left(s_{2}\right)\right)$.

Now we show that $x_{2}$ is less than 1:

$$
\begin{aligned}
x_{2} & <1, \\
-a_{1} & <1+a_{2}, \\
0 & <1+a_{1}+a_{2} .
\end{aligned}
$$

The last inequality can be proven by writing $1+a_{1}+a_{2}$ in the following way:

$$
\begin{aligned}
1+ & a_{1}+a_{2} \\
& =\underbrace{\left(1+\lambda_{1}\right)\left(1+\lambda_{2}\right)\left(1+\lambda_{3}\right)}_{>0}-\underbrace{\lambda_{1} \lambda_{2} \lambda_{3}}_{<0}>0 .
\end{aligned}
$$


Since $\lambda_{1} \lambda_{2} \lambda_{3}$ is $a_{0}$, we also get that $x_{3}$ is positive:

$$
x_{3}=\underbrace{\frac{-\overbrace{a_{0}}^{<0}}{1+a_{1}+a_{2}}}_{>0}>0 .
$$

Similarly, for the upper bound of $x_{3}$

$$
\begin{aligned}
x_{3} & <1, \\
-a_{0} & <1+a_{1}+a_{2}, \\
0 & <1+a_{0}+a_{1}+a_{2}, \\
0 & <\left(1+\lambda_{1}\right)\left(1+\lambda_{2}\right)\left(1+\lambda_{3}\right) .
\end{aligned}
$$

The vectors $b_{2}$ and $b_{3}$ are non-negative, because $(\mathbb{1}-G \mathbb{1})$ and $G(\mathbb{1}-G \mathbb{1})$ are the one-and two-step exit probability vectors of $\operatorname{DPH}(\gamma, \boldsymbol{G})$, and $0 \leq x_{2}, x_{3}<1$.

Finally, from the matrix equation $\boldsymbol{G} \boldsymbol{B}=\boldsymbol{B} \boldsymbol{A}$ we have an explicit expression for $b_{1}$,

$$
b_{1}=\frac{1}{\left(1-x_{1}\right)\left(1-x_{2}\right)\left(1-x_{3}\right)} \boldsymbol{G}^{2}(\mathbb{1}-\boldsymbol{G} \mathbb{1}) .
$$

That is, $b_{1}$ is a three-step exit probability vector multiplied by a positive constant.

Theorem 8 does not cover the case when $\left|s_{2}\right|^{2}>$ $2 s_{1}\left(-\operatorname{Re}\left(s_{2}\right)\right)$. This can occur only when $s_{2}$ and $s_{3}$ are complex conjugate eigenvalues. The following theorem applies in this case.

Theorem 9. If the eigenvalues of a third-order $\operatorname{DPH}(\gamma, \boldsymbol{G})$ are $1 \geq\left|s_{1}\right| \geq\left|s_{2}\right| \geq\left|s_{3}\right|, \operatorname{Re}\left(s_{1}\right)>0>$ $\operatorname{Re}\left(s_{3}\right) \geq \operatorname{Re}\left(s_{2}\right)$, and $\left|s_{2}\right|^{2}>2 s_{1}\left(-\operatorname{Re}\left(s_{2}\right)\right)$, then we use the same canonical form as in the case of PPP in Theorem 5 .

Proof. Similarly to the proof of Theorem 5, we need to introduce the procedure of Horváth and Telek (2009) in order to prove the theorem, which we omit here.

\section{Canonical representation of second-order DMAPs}

In this section we give a canonical form for $\operatorname{DMAP}(2)$ processes.

We use an approach similar to that in Section 3 . i.e., we prove that every $\operatorname{DRAP}(2)$ can be transformed to the introduced Markovian canonical form. We do this by choosing a set of the bounds of DRAP(2) and show that they are the tight bounds of the introduced $\operatorname{DMAP}(2)$ canonical form, which means that $\operatorname{DRAP}(2) \subseteq$ canonical $\operatorname{DMAP}(2)$, but by definition canonical $\operatorname{DMAP}(2) \subseteq \operatorname{DRAP}(2)$. Consequently, $\operatorname{DRAP}(2) \equiv$ canonical DMAP(2).

DRAP(2) processes are defined by four-parameters (Telek and Horváth, 2007), e.g., the first three-factorial moments of the stationary inter-arrival time distribution $\left(f_{1}, f_{2}, f_{3}\right)$, and the correlation parameter $(\gamma) . \quad \boldsymbol{D}_{0}$ and $\boldsymbol{D}_{1}$ of size $2 \times 2$ have a total of eight-elements (free parameters). The $\left(D_{0}+D_{1}\right) \mathbb{1}=\mathbb{1}$ constraint reduces the number of free parameters to six. If, additionally, two elements of the representation are set to zero, then the obtained (canonical) representation characterizes the process exactly with four parameters.

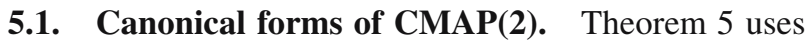
the relation of discrete and continuous distributions. We are going to utilize a similar relation between $\operatorname{DMAP}(2)$ and CMAP(2). To this end, we summarize the canonical representation of CMAP(2) from the work of Bodrog et al. (2008).

Theorem 10. (Bodrog et al., 2008) If the correlation parameter of a second-order $\operatorname{CRAP}\left(\boldsymbol{H}_{\mathbf{0}}, \boldsymbol{H}_{1}\right)$ is

- non-negative, then it can be represented in the following Markovian canonical form:

$$
\begin{aligned}
& \boldsymbol{D}_{\mathbf{0}}=\left[\begin{array}{cc}
-\lambda_{1} & (1-a) \lambda_{1} \\
0 & -\lambda_{2}
\end{array}\right], \\
& \boldsymbol{D}_{\mathbf{1}}=\left[\begin{array}{cc}
a \lambda_{1} & 0 \\
(1-b) \lambda_{2} & b \lambda_{2}
\end{array}\right] .
\end{aligned}
$$

where $0<\lambda_{1} \leq \lambda_{2}, 0 \leq a, b \leq 1, \min \{a, b\} \neq$ $1, \gamma=a b$, and the associated embedded stationary vector is

$$
\boldsymbol{\pi}=\left[\begin{array}{ll}
\frac{1-b}{1-a b} & \frac{b-a b}{1-a b}
\end{array}\right]
$$

- negative, then it can be represented in the following Markovian canonical form:

$$
\begin{aligned}
& \boldsymbol{D}_{\mathbf{0}}=\left[\begin{array}{cc}
-\lambda_{1} & (1-a) \lambda_{1} \\
0 & -\lambda_{2}
\end{array}\right], \\
& \boldsymbol{D}_{\mathbf{1}}=\left[\begin{array}{cc}
0 & a \lambda_{1} \\
b \lambda_{2} & (1-b) \lambda_{2}
\end{array}\right],
\end{aligned}
$$

where $0<\lambda_{1} \leq \lambda_{2}, 0 \leq a \leq 1,0<b \leq 1$, $\gamma=-a b$, and the associated embedded stationary vector is

$$
\boldsymbol{\pi}=\left[\frac{b}{1+a b} \quad 1-\frac{b}{1+a b}\right]
$$

\subsection{Canonical forms of $\operatorname{DMAP}(2)$ with positive eigen-} values.

Theorem 11. If the eigenvalues of $\boldsymbol{H}_{\mathbf{0}}$ are positive and the correlation parameter of a second-order $\operatorname{DRAP}\left(\boldsymbol{H}_{\mathbf{0}}, \boldsymbol{H}_{\mathbf{1}}\right)$ is 
- non-negative, then it can be represented in the following Markovian canonical form:

$$
\begin{aligned}
& \boldsymbol{D}_{\mathbf{0}}=\left[\begin{array}{cc}
1-\lambda_{1} & (1-a) \lambda_{1} \\
0 & 1-\lambda_{2}
\end{array}\right], \\
& \boldsymbol{D}_{\mathbf{1}}=\left[\begin{array}{cc}
a \lambda_{1} & 0 \\
(1-b) \lambda_{2} & b \lambda_{2}
\end{array}\right],
\end{aligned}
$$

where $0<\lambda_{1} \leq \lambda_{2}, 0 \leq a, b<1, \gamma=a b$, and the associated embedded stationary vector is

$$
\boldsymbol{\pi}=\left[\begin{array}{ll}
\frac{1-b}{1-a b} & \frac{b-a b}{1-a b}
\end{array}\right]
$$

- negative, then it can be represented in the following Markovian canonical form

$$
\begin{aligned}
& \boldsymbol{D}_{\mathbf{0}}=\left[\begin{array}{cc}
1-\lambda_{1} & (1-a) \lambda_{1} \\
0 & 1-\lambda_{2}
\end{array}\right], \\
& \boldsymbol{D}_{\mathbf{1}}=\left[\begin{array}{cc}
0 & a \lambda_{1} \\
b \lambda_{2} & (1-b) \lambda_{2}
\end{array}\right],
\end{aligned}
$$

where $0<\lambda_{1} \leq \lambda_{2}, s_{1}=1-\lambda_{1}, s_{2}=1-\lambda_{2}$, $0 \leq a \leq 1,0<b \leq 1, \gamma=-a b$, and the associated embedded stationary vector is

$$
\boldsymbol{\pi}=\left[\frac{b}{1+a b} \quad 1-\frac{b}{1+a b}\right] .
$$

Proof. Practically the same approach is applied here as in Theorem 5. First note that, if $\left(\boldsymbol{H}_{\mathbf{0}}, \boldsymbol{H}_{\mathbf{1}}\right)$ is a DRAP(2), then $\left(\boldsymbol{H}_{\mathbf{0}}-\boldsymbol{I}, \boldsymbol{H}_{\mathbf{1}}\right)$ is a $\operatorname{CRAP}(2)$. Using this,

$$
\begin{aligned}
\operatorname{DRAP} & \left(\boldsymbol{H}_{\mathbf{0}}, \boldsymbol{H}_{\mathbf{1}}\right) \\
& \stackrel{D}{\Rightarrow} C \operatorname{CRAP}\left(\boldsymbol{H}_{\mathbf{0}}-\boldsymbol{I}, \boldsymbol{H}_{\mathbf{1}}\right) \\
& \equiv \operatorname{CMAP}\left(\boldsymbol{T}^{-1}\left(\boldsymbol{H}_{\mathbf{0}}-\boldsymbol{I}\right) \boldsymbol{T}, \boldsymbol{T}^{-1}\left(\boldsymbol{H}_{\mathbf{1}}\right) \boldsymbol{T}\right)
\end{aligned}
$$

proves the theorem. The steps are self-explanatory, except for the equivalence in the above expression, which is based on Theorem 10 of Bodrog et al. (2008).

\subsection{Canonical forms of $\operatorname{DMAP}(2)$ with a negative eigenvalue.}

Theorem 12. If one eigenvalue of $\boldsymbol{H}_{\mathbf{0}}$ is negative and the correlation parameter of a second-order $\operatorname{DRAP}\left(\boldsymbol{H}_{\mathbf{0}}, \boldsymbol{H}_{\mathbf{1}}\right)$ is

- non-negative, then it can be represented in the following Markovian canonical form:

$$
\begin{aligned}
& \boldsymbol{D}_{\mathbf{0}}=\left[\begin{array}{cc}
1-\beta_{1} & a \beta_{1} \\
\frac{1}{a} \beta_{2} & 0
\end{array}\right], \\
& \boldsymbol{D}_{\mathbf{1}}=\left[\begin{array}{cc}
(1-a) \beta_{1} & 0 \\
\left(1-\frac{1}{a} \beta_{2}\right) b & \left(1-\frac{1}{a} \beta_{2}\right)(1-b)
\end{array}\right] ;
\end{aligned}
$$

- negative, then it can be represented in the following Markovian canonical form:

$$
\begin{aligned}
& \boldsymbol{D}_{\mathbf{0}}=\left[\begin{array}{cc}
1-\beta_{1} & a \beta_{1} \\
\frac{1}{a} \beta_{2} & 0
\end{array}\right], \\
& \boldsymbol{D}_{\mathbf{1}}=\left[\begin{array}{cc}
0 & (1-a) \beta_{1} \\
\left(1-\frac{1}{a} \beta_{2}\right) b & \left(1-\frac{1}{a} \beta_{2}\right)(1-b)
\end{array}\right],
\end{aligned}
$$

where the eigenvalues are such that $s_{2}<0<s_{1}<1$, $s_{1}+s_{2}>0$, the relation of the parameters and the eigenvalues is

$$
\begin{gathered}
\beta_{1}=1-s_{1}-s_{2}, \\
\beta_{2}=\frac{s_{1} s_{2}}{s_{1}+s_{2}-1}, 0 \leq b<1, \\
\beta_{2} \leq a \leq \min \left(1, b \frac{1-s_{2}}{1-s_{1}}\right)
\end{gathered}
$$

in the case of $\gamma \geq 0$ or $\beta_{2} \leq a \leq 1$ in the case of $\gamma<0$.

The correlation parameter and the first coordinate of the embedded stationary probability vectors (the unique solution of (5))

- of (54) are

$$
\begin{gathered}
\gamma=(1-a)(1-b)\left(1+\frac{1-a}{a} \frac{s_{1} s_{2}}{1-s_{1}-s_{2}+s_{1} s_{2}}\right) \\
\pi_{1}=\frac{1-\frac{1}{1-a} \gamma}{1-\gamma},
\end{gathered}
$$

- of 55) are

$$
\begin{gathered}
\gamma=-(1-a) b\left(1+\frac{1-a}{a} \frac{s_{1} s_{2}}{1-s_{1}-s_{2}+s_{1} s_{2}}\right) \\
\pi_{1}=1-\frac{1+\frac{a}{1-a} \gamma}{1-\gamma} .
\end{gathered}
$$

We prove the theorem by considering the full flexibility of the class DRAP(2) with a negative eigenvalue and showing that the canonical forms of Theorem 12 cover this whole set of processes. To this end, we first investigate the flexibility of the class $\operatorname{DRAP}(2)$.

5.3.1. Constraints of the class $\operatorname{DRAP}(2)$. We investigate the flexibility of the class DRAP(2) based on the following representation:

$$
\begin{aligned}
& \boldsymbol{H}_{\mathbf{0}}=\left[\begin{array}{cc}
s_{1} & 0 \\
0 & s_{2}
\end{array}\right], \\
& \boldsymbol{H}_{\mathbf{1}}=\left[\begin{array}{cc}
a_{1}+\left(1-a_{1}-s_{1}\right) \gamma & \left(1-a_{1}-s_{1}\right)(1-\gamma) \\
\frac{a_{1}\left(1-s_{2}\right)(1-\gamma)}{1-s_{1}} & \frac{\left(1-s_{2}\right)\left(1-a_{1}-s_{1}+a_{1} \gamma\right)}{1-s_{1}}
\end{array}\right],
\end{aligned}
$$


where $s_{1}$ is the positive and $s_{2}$ is the negative eigenvalue, $\gamma$ is the correlation parameter, and $a_{1}$ is the parameter that characterizes the stationary inter-arrival distribution together with the eigenvalues according to (13). With this representation the first coordinate of the embedded stationary vector is $\pi_{1}=a_{1} /\left(1-s_{1}\right)$.

For a given pair of eigenvalues, $s_{1}>0$ and $s_{2}<0$, Theorem 1 defines the limits of $a_{1}$. According to these limits, the first coordinate of any embedded vector of $\operatorname{DRAP}\left(\boldsymbol{H}_{\mathbf{0}}, \boldsymbol{H}_{1}\right)$ should be bounded by

$$
\frac{\left(1-s_{2}\right) s_{2}}{\left(1-s_{2}\right) s_{2}-\left(1-s_{1}\right) s_{1}} \leq x \leq \frac{\left(1-s_{2}\right)\left(1-s_{2}\right)}{s_{1}-s_{2}} .
$$

The function $U_{n}(x)$ describes the effect of an $n$ long inter-arrival period on the first coordinate of the embedded vector,

$$
U_{n}(x)=\frac{(x, 1-x) \boldsymbol{H}_{\mathbf{0}}{ }^{n-1} \boldsymbol{H}_{\mathbf{1}}}{(x, 1-x) \boldsymbol{H}_{\mathbf{0}}{ }^{n-1} \boldsymbol{H}_{\mathbf{1}} \mathbb{1}}(1,0)^{T} .
$$

If the embedded vector is $(x, 1-x)$ at an arrival instance and the next inter-arrival is $n$ time unit long, the embedded vector is going to be $\left(U_{n}(x), 1-U_{n}(x)\right)$ at the next arrival instance. In the case of DMAPs the embedded vector represents the probability distribution of the background Markov chain at arrivals, but in the case of DRAPs it does not have any probabilistic interpretations. $\boldsymbol{H}_{\mathbf{0}}$ and $\boldsymbol{H}_{\mathbf{1}}$ have to be such that starting from the stationary embedded vector $\pi$ for any series of inter-arrival times, the first coordinate of the embedded vector satisfies (61). Based on this property, we define simple constraints:

- Long series of one time unit long inter-arrivals: $U_{1}(x)=x$ has to have a real solution between the bounds in 61, because if the solution were complex or larger (smaller) than the respective bound, then a series of one time unit long inter-arrivals would increase (decrease) the first coordinate above the upper (below the lower) limit (cf. Fig. 1). This constraint results in

$$
\gamma \leq \frac{\left(\sqrt{c_{1}}-\sqrt{c_{2}}\right)^{2}}{\left(c_{3}-a_{1} s_{2}\right)^{2}} .
$$

- Long series of one time unit long inter-arrivals, then a two time units long inter-arrival: If $\gamma>0$, then $U_{1}(x)$ is a shifted negative hyperbolic function which increases monotonously between the bounds in (61). If $U_{1}(x)=x$ has two solutions, $w_{1}, w_{2}$ $\left(w_{1}<w_{2}\right)$, then $w_{1}$ is stable and $w_{2}$ is unstable, which means that, starting from $x<w_{1}$ or $w_{1}<$ $x<w_{2}$ and having a long series of one time unit long inter-arrivals, the first coordinate converges to $w_{1}$, while starting from $x>w_{2}$ and having a long series of one time unit long inter-arrivals, the first coordinate diverges. Consequently, a long series of one time unit long inter-arrivals and a two time units long inter-arrival keep the first coordinate between the bounds if $U_{2}\left(w_{1}\right) \leq w_{2}$. This constraint results in

$$
\begin{aligned}
\gamma \leq & \frac{s_{1} s_{2} c_{2}-c_{1}\left(1-s_{1}-s_{2}\right)}{c_{4} c_{5}} \\
& -\frac{\sqrt{s_{1} s_{2} c_{1} c_{2}\left(s_{1}+s_{2}\right)^{2}}}{c_{4} c_{5}}
\end{aligned}
$$

- Long series of two time units long inter-arrivals: Similarly to the first constraint, $U_{2}(x)=x$ has to have a real solution which results in

$$
\gamma \geq \frac{\left.\sqrt{s_{1} s_{2} c_{2}}+\sqrt{c_{6}}\right)^{2}}{c_{4}{ }^{2}}
$$

- Long series of one time unit long inter-arrivals: If $\gamma<0$, then $U_{1}(x)$ is a shifted hyperbolic function which decreases monotonously between the bounds in (61). $U_{1}(x)=x$ has to have a stable real solution $\left(w_{1}\right)$ between the bounds in (61), which holds if

$$
\left.\frac{\mathrm{d}}{\mathrm{d} x} U_{1}(x)\right|_{x=w_{1}}>-1
$$

(cf. Fig. 2) (in the case of a long series of 1 time unit long inter-arrivals the first coordinate converges to $w_{1}$ ). This constraint results in

$$
\gamma \geq \frac{s_{2}\left(1-a_{1}-s_{1}\right)+a_{1} s_{1}}{\left(c_{3}-a_{1} s_{1}\right)^{2}} .
$$

In the above expressions the auxiliary variables are

$$
\begin{aligned}
& c_{1}=-a_{1}\left(s_{1}-s_{2}\right)^{2}\left(1-a_{1}-s_{1}\right), \\
& c_{2}=\left(1-s_{1}\right)^{3}\left(1-s_{2}\right), \\
& c_{3}=1-s_{1}\left(2-a_{1}-s_{1}\right), \\
& c_{4}=s_{1}(1-s 1)\left(1-a_{1}-s_{1}\right)+a_{1} s_{2}\left(1-s_{2}\right), \\
& c_{5}=\left(a_{1}\left(s_{1}-s_{2}\right)+s_{2}\left(1-s_{1}\right)^{2}\right), \\
& c_{6}=-a_{1}\left(1-a_{1}-s_{1}\right)\left(s_{1}\left(1-s_{1}\right)-s_{2}\left(1-s_{2}\right)\right)^{2} .
\end{aligned}
$$

We summarize the results of this subsection in the following theorem.

Theorem 13. For DRAP $\left(\boldsymbol{H}_{\mathbf{0}}, \boldsymbol{H}_{\mathbf{1}}\right)$ defined in 60) with $0<s_{1}<1,-s_{1}<s_{2}<0$ and $a_{1}$ satisfying Theorem 1 the correlation parameter satisfies the inequalities (63)(66).

Theorem 13 defines only some bounds of the set of DRAP(2) processes, but the subsequent analysis of the canonical DMAP(2) proves that these bounds are tight. 


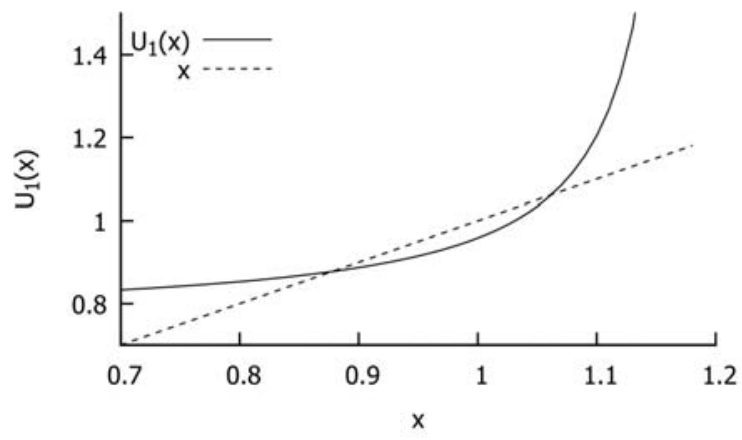

Fig. 1. $U_{1}(x)$ when $s_{1}=0.8, s_{2}=-0.3, a_{1}=0.19, \gamma=0.17$.

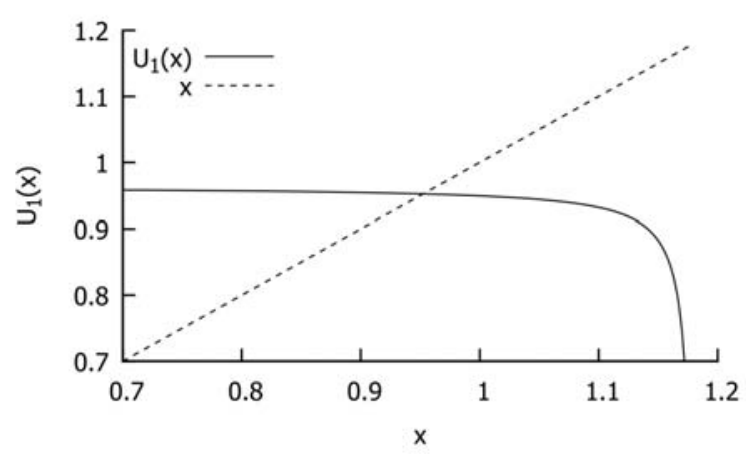

Fig. 2. $U_{1}(x)$ when $s_{1}=0.8, s_{2}=-0.3, a_{1}=0.19, \gamma=$ -0.012 .

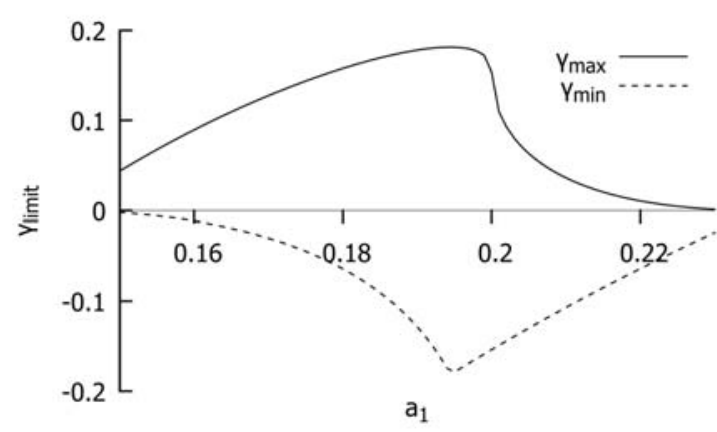

Fig. 3. Upper and lower $\gamma$ limits as a function of $a_{1}$ when $s_{1}=$ $0.8, s_{2}=-0.3$.

5.3.2. Constraints of the set of canonical DMAP(2) processes. Having the bounds of the $\operatorname{DRAP}(2)$ class from Theorem 13 we are ready to prove Theorem 12

Proof. (Theorem 12) First we need to relate the variables of the canonical representation with the parameters used for characterizing DMAP(2) processes. The relation of $\beta_{1}, \beta_{2}$ with $s_{1}, s_{2}$ is

$$
s_{1,2}=\frac{1}{2}\left(1-\beta_{1} \pm \sqrt{\left(1-\beta_{1}\right)^{2}+4 \beta_{1} \beta_{2}}\right) .
$$

The relation of $s_{1}, s_{2}, a_{1}, \gamma$ with $a$ and $b$ can be obtained from (56) and (57) for the first canonical form and from (58) and (59) for the second canonical form.

$$
\begin{aligned}
& \text { If } \gamma>0, \text { then } \\
& \begin{aligned}
a & =\frac{g_{1}+\sqrt{g_{1}^{2}-g_{2}}}{2 e_{1}}, \\
b & =1-\frac{a \gamma\left(1-s_{1}-s_{2}+s_{1} s_{2}\right)}{(1-a)\left(a\left(1-s_{1}-s_{2}\right)+s_{1} s_{2}\right)},
\end{aligned}
\end{aligned}
$$

where

$$
\begin{aligned}
& e_{1}=\left(1-s_{1}\right)\left(1-s_{1}-s_{2}\right)^{2}, \\
& e_{2}=\left(1-s_{1}-s_{2}\right)\left(a_{1}\left(s_{1}-s_{2}\right)(1-\gamma)-s_{1}\left(1-s_{1}\right)\right), \\
& e_{3}=\gamma\left(1-s_{1}\right)^{2} \\
& g_{1}=e_{1}+e_{2}-e_{3}\left(1-s_{1}-s_{2}\right), \\
& g_{2}=4 e_{1}\left(e_{2}+e_{3} s_{1}\right),
\end{aligned}
$$

and if $\gamma<0$, then

$$
\begin{aligned}
& a=\frac{g_{3}-\sqrt{g_{3}^{2}+g_{4}}}{g_{5}}, \\
& b=1-\frac{a \gamma\left(1-s_{1}-s_{2}-s_{1} s_{2}\right)}{(1-a)\left(a\left(1-s_{1}-s_{2}\right)+s_{1} s_{2}\right)},
\end{aligned}
$$

where

$$
\begin{aligned}
e_{6}= & a_{1}(s 1-s 2)(1-\gamma), \\
e_{7}= & \left(1-s_{1}\right)\left(s_{2}(1-\gamma)-\left(1-s_{1}-s_{2}\right) \gamma\right), \\
& e_{8}=\left(1-s_{1}-s_{2}\right)\left(1-s_{1}\right) s_{2}, \\
g_{3} & =-\left(1-s_{1}-s_{2}\right) e_{6}+e_{7} s_{1}-e_{8}, \\
g_{4} & =4\left(e_{6}+e_{7}\right) e_{8} s_{1}, \\
& g_{5}=-2\left(1-s_{1}-s_{2}\right)\left(e_{6}+e_{7}\right) .
\end{aligned}
$$

Based on these relations, the constraints of the canonical DMAP(2) processes can be obtained using the fact that all the elements of $D_{0}$ and $D_{1}$ have to be non-negative real numbers. That is, $a$ is real, $\beta_{2} \leq a \leq 1$ and $0 \leq b \leq$ 1. The parameter $a$ is real when the expression under the square root sign in (69) for $\gamma>0$ and in (71) for $\gamma<0$ is non-negative. Altogether, these constrains result in five inequalities for $\gamma>0$ and five for $\gamma<0$. Out of these, the following are relevant:

- Case $\gamma>0$ :

- $a$ is real when $g_{1}^{2}-g_{2} \geq 0$, which translates to 63.

- the inequality $b \leq 1$ translates to 64.

- Case $\gamma<0$ :

- $a$ is real when $g_{3}^{2}+g_{4} \geq 0$, which translates to (65),

- the inequality $b \geq 0$ translates to 66 .

The appendix provides a detailed derivation of (63) based on $g_{1}^{2}-g_{2} \geq 0$. We neglect the details of the other derivations. 


\section{Explicit moments and correlation matching with the canonical forms}

One of the most important applications of the introduced canonical forms is the factorial moment matching for $\mathrm{DPH}(2)$ and $\mathrm{DPH}(3)$ distributions as well as the factorial moment and correlation matching of DMAP(2) processes.

In the second part of this section we give explicit factorial moment and correlation matching formulas for second-order models. While such formulas cannot be provided for $\mathrm{DPH}(3)$, the canonical form still makes moment matching possible. In the first part of this section we discuss this matching procedure for DPH distributions in general.

6.1. Moment matching with DPH. To obtain formulae for moment matching, the inverse of (3) is required, that is, we need to find a vector-matrix pair based on a given set of factorial moments. For a full characterization of a $\operatorname{DPH}(n)$, we need the first $2 n-$ 1 factorial moments $\left(f_{1}, f_{2}, \ldots, f_{2 n-1}\right)$. We find an appropriate vector-matrix pair exhibiting a given set of factorial moments using the procedure available for $\mathrm{CPH}$ moment matching of Horváth and Telek (2007). In spite of the fact that (10) and (3) look similar, we cannot directly use the $\mathrm{CPH}$ moment matching method for DPH moment matching, because of the extra term in (3). That is why we first transform the factorial moments such that they exhibit an expression similar to (10).

Let us define $\boldsymbol{G}=-(\boldsymbol{I}-\boldsymbol{A})^{-1} \boldsymbol{A}$. Then $\boldsymbol{A}^{-1}=$ $\boldsymbol{I}-\boldsymbol{G}^{-1}$. Substituting this into (3), we get

$$
\begin{aligned}
\frac{f_{i}}{i !} & =\alpha\left((\boldsymbol{I}-\boldsymbol{A})^{-1} \boldsymbol{A}\right)^{i} \boldsymbol{A}^{-1} \mathbb{1} \\
& =\alpha(-\boldsymbol{G})^{i}\left(\boldsymbol{I}-\boldsymbol{G}^{-1}\right) \mathbb{1} \\
& =(-1)^{i} \alpha\left(\boldsymbol{G}^{i}-\boldsymbol{G}^{i-1}\right) \mathbb{1} .
\end{aligned}
$$

Assuming $f_{0}=1$ and $\alpha \mathbb{1}=1$, from (73) we have

$$
\sum_{i=0}^{k}(-1)^{i} \frac{f_{i}}{i !}=\alpha G^{k} \mathbb{1} .
$$

Multiplying both the sides by $k$ !, we obtain

$$
\hat{\mu}_{k} \triangleq k ! \sum_{i=0}^{k}(-1)^{i} \frac{f_{i}}{i !}=k ! \alpha G^{k} \mathbb{1}
$$

which has the same form as 10 . Applying the $\mathrm{CPH}$ moment matching procedure with $\hat{\mu}_{k}$ results in $\alpha$ and $\boldsymbol{G}$ which satisfy (75). Finally, the matrix $\boldsymbol{A}$ is obtained from $\boldsymbol{A}=(\boldsymbol{G}-\boldsymbol{I})^{-1} \boldsymbol{G}$. This procedure commonly generates a non-Markovian matrix $\boldsymbol{A}$.

6.1.1. Moment matching with canonical $\operatorname{DPH}(2)$ and DPH(3). Applying the general DPH moment matching procedure of the previous subsection, we attain an $(\alpha, \boldsymbol{A})$ $\operatorname{MG}(2)$ or $\mathrm{MG}(3)$ representation based on $\left(f_{1}, f_{2}, f_{3}\right)$ or $\left(f_{1}, f_{2}, \ldots, f_{5}\right)$. By determining the eigenvalues of $\boldsymbol{A}$, the appropriate type of canonical form can be decided and its elements can be calculated according to Sections 3 or 4 , If the resulting representation is Markovian, then the given set of factorial moments can be matched with a $\mathrm{DPH}(2)$ or a $\mathrm{DPH}(3)$. Otherwise, it is not possible.

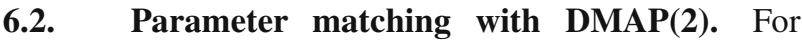
DMAP(2) processes, the previously mentioned inverse characterization is possible, that is, the first three moments $\left(f_{1}, f_{2}, f_{3}\right)$ and the correlation parameter $(\gamma)$ can be used to give explicit formulae for $\beta_{1}, \beta_{2}, a, b$ of Theorems 11 and 12 .

Our matching method is composed of two steps. The first one is moment matching with a DPH(2). The result of this phase is an $(\alpha, \boldsymbol{A})$ canonical $\mathrm{DPH}(2)$ representation. The second one is the matching of $\gamma$. This means the calculation of $a$ and $b$ of Theorems 11 and 12 from $\alpha$, $\boldsymbol{A}$, and $\gamma$.

6.2.1. Bounds of DMAP(2) processes. For exact parameter matching, first it has to be decided if a DMAP(2) exists with a given set of $f_{1}, f_{2}, f_{3}, \gamma$ moments and correlation parameter set, and if the matching is possible, it has to be determined if one of the eigenvalues of $D_{0}$ is negative, as this affects the formulae for the elements of the canonical form. To this end, moment and a correlation bounds have to be established.

It can be easily proven that the class of $\mathrm{DPH}(2)$ distributions can be defined as the stationary inter-arrival time distribution of DMAP(2) processes. Thus, their moment bounds are identical. These bounds can be derived from the Markovian constraints on the canonical form of DPH(2) distributions (i.e., the elements of $\alpha$ and $\boldsymbol{A}$ in Theorems 24 have to be between 0 and 1). For $\boldsymbol{A}$ with two positive eigenvalues, the constraints are already given by Telek and Heindl (2002). These results are summarized in Table 1, where

$$
\begin{aligned}
j_{1}= & \frac{6}{\left(2 f_{1}+\sqrt{2 j_{2}}\right)^{3}}\left(f_{1}\left(2 f_{1}+\sqrt{2 j_{2}}\right)\left(3 f_{2}+2 f_{1}\right)\right. \\
& \left.\times\left(f_{2}-2 f_{1}+2\right)-2 f_{2}^{2}\left(f_{2}-\sqrt{2 j_{2}}\right)\right),
\end{aligned}
$$

and $j_{2}=2 f_{1}^{2}-2 f_{1}-f_{2}$.

For the negative eigenvalue case, we have derived similar constraints as shown in Table 2] where

$$
j_{3}=\frac{3 \sqrt{\left(f_{2}-2 f_{1}\left(f_{1}-5\right)-8\right)\left(f_{2}-2 f_{1}\left(f_{1}-1\right)\right)^{3}}}{4\left(f_{1}-1\right)}
$$




$$
\begin{aligned}
& +\frac{3\left(-4 f_{1}\left(f_{1}-2\right)\left(f_{1}-1\right)^{2}+8 f_{2}+4 f_{1} f_{2}\left(f_{1}-3\right)\right)}{4\left(f_{1}-1\right)} \\
& +\frac{f_{2}^{2}}{4\left(f_{1}-1\right)} .
\end{aligned}
$$

In the following we present formulae for $\beta_{1}, \beta_{2}, p$. Substituting them into Eqns. (63)-(66), exact $\gamma$ bounds can be easily derived. However, the resulting expressions are rather long, and therefore we do not show them.

6.2.2. Transformation to the $\operatorname{DMAP}(2)$ canonical form with positive eigenvalues. If the $f_{1}, f_{2}, f_{3}$ moments are in the bounds described by Table 1 they can be matched with a $\mathrm{DPH}(2)$ with positive eigenvalues. In this case the first step is based on Table 3 by Telek and Heindl (2002). The $s_{1}$ and $s_{2}$ elements of matrix $\boldsymbol{A}$ and vector $\alpha$ can be calculated as

$$
\begin{aligned}
\alpha & =[p, 1-p], \\
p & =\frac{-z\left(h_{3}-6 f_{1} h_{1}\right)+\sqrt{h_{4}}}{z h_{3}+\sqrt{h_{4}}}, \\
s_{1} & =1-\frac{h_{3}-z \sqrt{h_{4}}}{h_{2}}, \\
s_{2} & =1-\frac{h_{3}+z \sqrt{h_{4}}}{h_{2}},
\end{aligned}
$$

where

$$
\begin{aligned}
h_{1} & =2 f_{1}{ }^{2}-2 f_{1}-f_{2}, h_{2}=3 f_{2}{ }^{2}-2 f_{1} f_{3}, \\
h_{3} & =3 f_{1} f_{2}-6\left(f_{1}+f_{2}-f_{1}^{2}\right)-f_{3}, \\
h_{4} & =h_{3}^{2}-6 h_{1} h_{2}, \\
z & =\frac{h_{2}}{\left|h_{2}\right|} .
\end{aligned}
$$

The second step is the calculation of $a, b$ of Theorem 11 . If $\gamma=0$, then $a=1, b=0$. If $\gamma>0$, then $a$ and $b$ can be computed using

$$
a=\frac{d_{1}-\sqrt{d_{2}}}{2\left(1-s_{1}\right)}, \quad b=\frac{d_{1}+\sqrt{d_{2}}}{2\left(1-s_{2}\right)},
$$

with

$$
\begin{aligned}
d_{1} & =1-s_{2}-p\left(1-s_{2}\right)(1-\gamma)+\left(1-s_{1}\right) \gamma, \\
d_{2} & =d_{1}^{2}-4\left(1-s_{1}\right)\left(1-s_{2}\right) \gamma .
\end{aligned}
$$

If $\gamma \leq 0$, then

$$
\begin{aligned}
a & =\frac{-\gamma\left(1-s_{2}\right)}{p\left(1-s_{2}\right)(1-\gamma)-\gamma\left(1-s_{1}\right)}, \\
b & =\frac{p\left(1-s_{2}\right)(1-\gamma)-\gamma\left(1-s_{1}\right)}{1-s_{2}} .
\end{aligned}
$$

6.2.3. Transformation to the canonical form with a negative eigenvalue. If the $f_{1}, f_{2}, f_{3}$ moments are in the bounds described by Table 2 , they can be matched with a DPH(2) with a positive and a negative eigenvalue. In this case the parameters $\beta_{1}, \beta_{2}$ and the vector $\alpha$ can be computed using

$$
\begin{aligned}
\beta_{1} & =\frac{12 f_{1}^{2}-3 f_{2}\left(4+f_{2}\right)-2 f_{3}+2 f_{1}\left(-6+3 f_{2}+f_{3}\right)}{\left(3 f_{2}^{2}-2 f_{1} f_{3}\right)}, \\
\beta_{2} & =\frac{-3 f_{2}\left(2-2 f_{1}+f_{2}\right)+2\left(-1+f_{1}\right) f_{3}}{12 f_{1}^{2}-3 f_{2}\left(4+f_{2}\right)-2 f_{3}+2 f_{1}\left(-6+3 f_{2}+f_{3}\right)}, \\
p & =\frac{\beta_{1}-f_{1} \beta_{1}+\beta_{2}+f_{1} \beta_{1} \beta_{2}}{-1+\beta_{2}}, \quad \alpha=[p, 1-p] .
\end{aligned}
$$

From $\beta_{1}$ and $\beta_{2}$, the eigenvalues $s_{1}$ and $s_{2}$ are obtained by (68). In the second step, $a$ and $b$ of Theorem 12 are calculated. If $\gamma=0$, then $a=1$ and $b=0$ stand again. Otherwise, if $\gamma>0$, then

$$
\begin{aligned}
& a=\frac{k_{1}+\sqrt{k_{1}^{2}-k_{2}}}{2 \beta_{1}}, \\
& b=1-\frac{a \gamma\left(1-\beta_{2}\right)}{(1-a)\left(a-\beta_{2}\right)},
\end{aligned}
$$

and if $\gamma<0$, then

$$
\begin{aligned}
& a=\frac{k_{3}+\sqrt{k_{3}^{2}+4 \beta_{2} k_{4}}}{2 k_{4}}, \\
& b=-\frac{a \gamma\left(1-\beta_{2}\right)}{(1-a)\left(a-\beta_{2}\right)},
\end{aligned}
$$

where

$$
\begin{aligned}
& k_{1}=(1-\gamma)\left(p+\beta_{1}+\beta_{2}-p \beta_{2}\right)-1+\beta_{1}, \\
& k_{2}=4 \beta_{1}\left(k_{1}-\beta_{1}+\gamma-\beta_{2} \gamma\right), \\
& k_{3}=(1-\gamma)\left(-p\left(1-\beta_{2}\right)-2 \beta_{2}\right)-\gamma\left(1-\beta_{1}\right), \\
& k_{4}=k_{3}+\beta_{2}+\gamma-\beta_{2} \gamma .
\end{aligned}
$$

If the moments $f_{1}, f_{2}, f_{3}$ are out of the bounds described by Tables 1 and 2 , then exact matching is not possible.

\section{Fitting using canonical forms}

In some cases, fitting based on a well-chosen distance measure might capture important characteristics of traffic traces better than moment matching. Employing canonical forms is beneficial in this case, as well.

The main advantage of using canonical forms in model fitting compared with the corresponding general form is that, while the canonical forms have the full flexibility of the given class, the number of parameters that has to be optimized is lower. When fitting with DPH(2), the canonical form has three parameters instead of the five of the general form (DPH(2) has six elements and the 
Table 1. Bounds for the first three moments of DPH(2) distributions with positive eigenvalues

\begin{tabular}{|c|c|c|}
\hline condition & bounds & $\mathrm{DPH}(2)$ \\
\hline & $1 \leq f_{1}<\infty$ & - \\
\hline $1 \leq f_{1}<2$ & $2\left(f_{1}-1\right) \leq f_{2}<\infty$ & - \\
\hline $2 \leq f_{1}$ & $\frac{f_{1}\left(3 f_{1}-4\right)}{2} \leq f_{2}<\infty$ & - \\
\hline \multicolumn{3}{|c|}{$1 \leq f_{1}<2$} \\
\hline $2\left(f_{1}-1\right) \leq f_{2}$ & $j_{1} \leq f_{3}$ & $\beta_{1}=\beta_{2}$ \\
\hline$f_{2}<2 f_{1}\left(f_{1}-1\right)$ & $f_{3} \leq \frac{3 f_{2}\left(f_{2}-2 f_{1}+2\right)}{2\left(f_{1}-1\right)}$ & $\beta_{2}=1$ \\
\hline \multicolumn{3}{|c|}{$2 \leq f_{1}$} \\
\hline$\frac{f_{1}\left(3 f_{1}-4\right)}{2} \leq f_{2}$ & $j_{1} \leq f_{3}$ & $\beta_{1}=\beta_{2}$ \\
\hline$f_{2}<2\left(f_{1}-1\right)$ & $f_{3} \leq 6\left(f_{1}-1\right)\left(f_{2}-f_{1}^{2}+f_{1}\right)$ & $p=1$ \\
\hline $2\left(f_{1}-1\right) \leq f_{2}$ & $j_{1} \leq f_{3}$ & $\beta_{1}=\beta_{2}$ \\
\hline$f_{2}<1-\frac{1}{f_{1}}$ & $f_{3} \leq \frac{3 f_{2}\left(f_{2}-2 f_{1}+2\right)}{2\left(f_{1}-1\right)}$ & $\beta_{2}=1$ \\
\hline \multicolumn{3}{|c|}{$1 \leq f_{1}$} \\
\hline $2 f_{1}\left(f_{1}-1\right) \leq f_{2}$ & $\frac{3 f_{2}\left(f_{2}-2 f_{1}+2\right)}{2\left(f_{1}-1\right)} \leq f_{3}$ & $\beta_{2}=1$ \\
\hline
\end{tabular}

equation $\alpha \mathbb{1}=1$ gives one constraint). The canonical form of $\mathrm{DPH}(3)$ has five parameters instead of the eight of the general case (a DPH(3) has nine elements and $\alpha \mathbb{1}=1$ gives a single constraint again). Finally, canonical DMAP(2) has four parameters instead of six (DMAP(2) has eight elements, but the $\left(\boldsymbol{D}_{0}+\boldsymbol{D}_{\mathbf{1}}\right) \mathbb{1}=\mathbb{1}$ equation means two constraints). Having fewer parameters results in a faster and better fitting in general (for the chosen distance measure).

In this section we provide numerical examples to demonstrate the advantages of using canonical forms. We use DPH(3) fitting as an illustration. Our choice is motivated by the fact that $\mathrm{DPH}(3)$ s are significantly more complex than DPH(2)s. However, we can use a very straightforward fitting method for them with relative entropy as a distance measure, which makes the demonstration simpler than it would be with DMAP(2) fitting.

As mentioned above, we use relative entropy as a distance function in our examples. Having the $\mathcal{X}$ and $\mathcal{Y}$ discrete distributions on $\mathbb{N}^{+}$with pmfs $f(i)$ and $g(i)$, we can calculate their $H$ relative entropy (or
Table 2. Bounds for the first three moments of DPH(2) distributions with a negative eigenvalue

\begin{tabular}{|c|c|c|}
\hline condition & bounds & $\mathrm{DPH}(2)$ \\
\hline & $1 \leq f_{1}<\infty$ & - \\
\hline $1 \leq f_{1}<2$ & $2\left(f_{1}-1\right) \leq f_{2}<\infty$ & - \\
\hline $2 \leq f_{1}$ & $2\left(f_{1}-1\right)^{2} \leq f_{2}<\infty$ & - \\
\hline \multicolumn{3}{|c|}{$1 \leq f_{1}<2$} \\
\hline $\begin{array}{c}2\left(f_{1}-1\right) \leq f_{2} \\
f_{2}<2 f_{1}\left(f_{1}-1\right)\end{array}$ & $\begin{array}{l}\frac{3\left(f_{2}-2 f_{1}+2\right)\left(f_{1}+f_{2}\right)}{\left(2 f_{1}-1\right)} \leq f_{3} \\
f_{3} \leq \frac{3 f_{2}\left(f_{2}-2 f_{1}+2\right)}{2\left(f_{1}-1\right)}\end{array}$ & $\begin{array}{l}\beta_{2}=0 \\
\beta_{1}=1\end{array}$ \\
\hline \multicolumn{3}{|c|}{$2 \leq f_{1}$} \\
\hline $2\left(f_{1}-1\right)^{2} \leq f_{2}$ & $\frac{3 f_{2}\left(f_{2}-2 f_{1}+2\right)}{2\left(f_{1}-1\right)} \leq f_{3}$ & $\beta_{2}=0$ \\
\hline$f_{2}<f_{1}\left(2 f_{1}-3\right)$ & $f_{3} \leq 6\left(f_{1}-1\right)\left(f_{2}-f_{1}^{2}+f_{1}\right)$ & $p=1$ \\
\hline$f_{1}\left(2 f_{1}-3\right) \leq f_{2}$ & $\frac{3 f_{2}\left(f_{2}-2 f_{1}+2\right)}{2\left(f_{1}-1\right)} \leq f_{3}$ & $\beta_{2}=0$ \\
\hline$f_{2}<2 f_{1}(f 1-1)$ & $f_{3} \leq \frac{3\left(f_{2}-2 f_{1}+2\right)\left(f_{1}+f_{2}\right)}{\left(2 f_{1}-1\right)}$ & $\beta_{1}=1$ \\
\hline \multicolumn{3}{|c|}{$1 \leq f_{1}$} \\
\hline $1-2 f_{1}(f 1-1) \leq f_{2}$ & $\begin{array}{c}j_{3} \leq f_{3} \\
f_{3} \leq \frac{3 f_{2}\left(f_{2}-2 f_{1}+2\right)}{2\left(f_{1}-1\right)}\end{array}$ & $\begin{array}{l}p=0 \\
\beta_{2}=0\end{array}$ \\
\hline
\end{tabular}

Kullback-Leibler divergence) as

$$
H(\mathcal{X}, \mathcal{Y})=-\sum_{i=1}^{\infty} f(i) \ln \left(\frac{g(i)}{f(i)}\right)
$$

If $f(i)$ is zero for a given $i$, that part of the expression is considered zero. The relative entropy of two distributions is strictly non-negative and is only zero if $f(i)=g(i)$. Intuitively, higher $H$ means a bigger difference between the two distributions and a worse fitting in our case.

In the following we present the results of fitting to three different distributions. The first one is the discrete uniform distribution on 1 to 50 (i.e., $f(i)=0.02$ if $i=$ $1, \ldots, 50$ and $f(i)=0$ otherwise). The second one is $\mathrm{DPH}(4)$ with

$$
\boldsymbol{A}=\left[\begin{array}{cccc}
0.6 & 0.1 & 0.07 & 0.03 \\
0.3 & 0.06 & 0.22 & 0.36 \\
0.14 & 0.4 & 0.1 & 0.2 \\
0.3 & 0.1 & 0.2 & 0.05
\end{array}\right],
$$


which has a monotonically decreasing pmf, and the third one is $\operatorname{DPH}(3)$ with

$$
\alpha=[0.3,0.1,0.6], \quad \boldsymbol{A}=\left[\begin{array}{ccc}
0.2 & 0.75 & 0.05 \\
0.5 & 0.1 & 0.4 \\
0.1 & 0.7 & 0.07
\end{array}\right],
$$

which has a fluctuating pmf with a slow decay. We made the fitting using the built-in optimization function of Wolfram Mathematica (called NMinimize).

For the general form, we had to consider only one type of representation. In the case of the canonical form, we ran the fitting algorithm for all the different types of representations and chose the best one. When fitting the uniform distribution, we took the theoretical pmf values. In the other two cases we simulated 100,000 inter-arrival times using the respective $(\alpha, \boldsymbol{A})$ and fitted using the empirical pmf of the traces. The results are summarized in Table 3 They clearly show that canonical forms perform better than the general form in fitting. An intuitive explanation is that the canonical forms have fewer parameters, and consequently, the optimization is a simpler task than in the general case. Furthermore, as different representations describe the same distribution in the general form, they have the same distance from the fitted trace. This suggests that the relative entropy is a very bumpy function of the parameters for the general case, which also makes the optimization harder.

The uniform distribution was hard to fit for both the canonical and the general form. However, the former still gave a much better result in terms of both the distance and the running time. Similarly, both the canonical and the general form were able to fit the trace of DPH(4), but the canonical fitting was faster again. Probably the most interesting example is the fitting of the $\mathrm{DPH}(3)$ trace. The pmfs of the fitted DPHs can be seen in Fig. 4. While, in theory, a perfect fit would have been possible, the general form provided a poor solution. Using the canonical form resulted in a good fitting, although it took a long time. This is due to the slow decay of the distribution, because it makes the goal function much more complex as it has more elements than in the previous cases.

\section{Conclusions}

In this paper we presented canonical representations for second-order and third-order DPH distributions and second-order DMAPs. We provided a detailed proof for the validity of these canonical representations, gave explicit methods to obtain these representations, and proved that the second-order Markovian models are equivalent to their non-Markovian counterparts.

We demonstrated the benefits of these canonical forms in parameter matching and trace fitting. Using
Table 3. Fitting of distributions with the general and canonical $\mathrm{DPH}(3)$ form.

\begin{tabular}{|c|c|c|c|c|}
\hline \multirow{2}{*}{ Distribution } & \multicolumn{2}{|c|}{ General form } & \multicolumn{2}{c|}{ Canonical form } \\
\cline { 2 - 5 } & Distance & Time & Distance & Time \\
\hline \hline Uniform & 4.55 & $473 \mathrm{~s}$ & 0.355 & $168 \mathrm{~s}$ \\
\hline DPH(4) & 0.00256 & $511 \mathrm{~s}$ & $3.29 \times 10^{-4}$ & $319 \mathrm{~s}$ \\
\hline DPH(3) & 6.32 & $4859 \mathrm{~s}$ & 0.025 & $1571 \mathrm{~s}$ \\
\hline
\end{tabular}

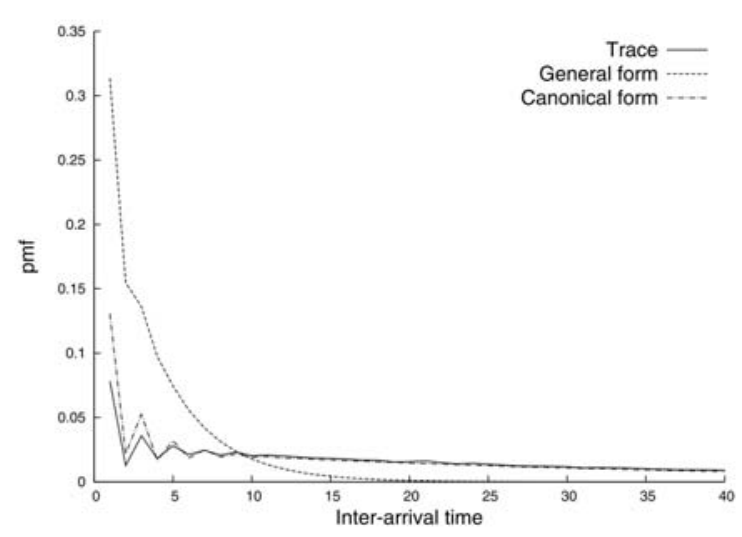

Fig. 4. Fitting of $\mathrm{DPH}(3)$ distribution with the general and canonical form.

them, we derived the moment and correlation bounds of second-order DMAPs (and DPHs), and presented explicit matching formulas for these parameters. For third-order DPH distributions we provided a simple procedure that can be used for moment matching.

We illustrated the advantages of fitting with canonical forms instead of the general form through numerical examples. The results confirmed that with canonical forms a substantially better performance can be achieved in both running time and fitting quality than with using general Markovian forms.

\section{Acknowledgment}

The authors gratefully acknowledge the support of the TÁMOP-4.2.2C-11/1/KONV-2012-0001 and OTKA K101150 projects. They would also like to thank Illés Horváth for his assistance in the proofs.

\section{References}

Alfa, A. (2002). Discrete time queues and matrix-analytic methods, Top 10(2): 147-185.

Bodrog, L., Heindl, A., Horváth, G. and Telek, M. (2008). A Markovian canonical form of second-order matrix-exponential processes, European Journal of Operation Research 190: 459-477. 
Horváth, G. and Telek, M. (2007). A canonical representation of order 3 phase type distributions, in K. Volter (Ed.), Formal Methods and Stochastic Models for Performance Evaluation, Springer, Berlin/Heidelberg, pp. 48-62.

Horváth, G. and Telek, M. (2009). On the canonical representation of phase type distributions, Performance Evaluation 66(8): 396-409.

Lakatos, L., Szeidl, L. and Telek, M. (2013). Introduction to Queueing Systems with Telecommunication Applications, Springer, New York, NY.

Neuts, M. (1981). Matrix Geometric Solutions in Stochastic Models, Johns Hopkins University Press, Baltimore, MD.

Telek, M. and Heindl, A. (2002). Matching moments for acyclic discrete and continuous phase-type distributions of second order, International Journal of Simulation Systems, Science \& Technology 3(3-4): 47-57.

Telek, M. and Horváth, G. (2007). A minimal representation of Markov arrival processes and a moments matching method, Performance Evaluation 64(9-12): 1153-1168.

András Mészáros is a Ph.D. student at the Department of Networked Systems and Services at the Budapest University of Technology and Economics. He received the M.Sc. degree in electrical engineering from the Technical University of Budapest in 2011. He carries out research in Markovian modelling of complex stochastic systems.

János Papp is an M.Sc. student at the Department of Mathematics of the Technical University of Budapest. He received the B.Sc. degree in mathematics from the same university in 2012. His research interest includes the canonical representation of $\mathrm{PH}$ distributions.

Miklós Telek received the M.Sc. degree in electrical engineering from the Technical University of Budapest in 1987. In the same year he joined the Hungarian Post Research Institute, where he studied the modelling, analysis and planning aspects of communication networks. Since 1990 he has been with the Department of Networked Systems and Services of the Technical University of Budapest, where he is a full professor now. He received the C.Sc. and D.Sc. degrees from the Hungarian Academy of Sciences in 1995 and 2004, respectively. His current research interest includes stochastic performance modelling as well as analysis of computer and communication systems.

\section{Appendix}

The constraint for the correlation coefficient is the solution of $g_{1}^{2}(\gamma)-g_{2}(\gamma)=0$ for $\gamma$. By substituting (70) into $g_{1}$ and $g_{2}$, regrouping the terms according to the different powers of $\gamma$, and simplifying the expression, we get

$$
\begin{aligned}
g_{1}^{2} & -g_{2} \\
= & \left(1-s_{1}-s_{2}\right)^{2}\left(1-s_{1}\left(2-a_{1}-s_{1}\right)-a_{1}-s_{2}\right)^{2} \gamma^{2} \\
& -2\left(1-s 1-s_{2}\right)^{2}\left[1+s_{1}^{2}\left(3-a_{1}\left(1-a_{1}+2 s_{2}\right)-3 s_{2}\right)\right. \\
& -s_{1}^{3}\left(1-a_{1}-s_{2}\right)-s_{2}\left(1+\left(1-a_{1}\right) a_{1} s_{2}\right) \\
& \left.-s_{1}\left(3-s_{2}\left(3+a_{1}\left(2-2 a_{1}+s_{2}\right)\right)\right)\right] \gamma \\
& +\left(1-s_{1}-s_{2}\right)^{2}\left(1-s_{1}\left(1+a_{1}-s_{2}\right)-s_{2}+a_{1} s_{2}\right)^{2} .
\end{aligned}
$$

By solving the equation $g_{1}^{2}(\gamma)-g_{2}(\gamma)=0$ and taking the smaller solution and simplifying the result, we get

$$
\begin{aligned}
\gamma & \frac{a_{1}^{2}\left(s_{1}-s_{2}\right)^{2}-a_{1}\left(1-s_{1}\right)\left(s_{1}-s_{2}\right)^{2}+\left(1-s_{1}\right)^{3}\left(1-s_{2}\right)}{\left(1-s_{1}\left(2-a_{1}-s_{1}\right)-a_{1} s_{2}\right)^{2}} \\
& -\frac{2 \sqrt{-a_{1}(1-s 1)^{3}\left(1-a_{1}-s_{1}\right)\left(s_{1}-s_{2}\right)^{2}\left(1-s_{2}\right)}}{\left(1-s_{1}\left(2-a_{1}-s_{1}\right)-a_{1} s_{2}\right)^{2}} .
\end{aligned}
$$

From this last expression, one can see that the numerator is the square of $\sqrt{-a_{1}\left(s_{1}-s_{2}\right)^{2}\left(1-a_{1}-s_{1}\right)}-$ $\sqrt{\left(1-s_{1}\right)^{3}\left(1-s_{2}\right)}$. The constraint will be this smaller solution. Finally, we get 63 by substituting the $c_{i}$ formulae from 67).

Received: 20 August 2013

Revised: 10 February 2014

Re-revised: 26 February 2014 